Xiaochun Liu' / Richard Luger ${ }^{2}$

\title{
Markov-switching quantile autoregression: a Gibbs sampling approach
}

\author{
${ }^{1}$ Department of Economics, Finance and Legal Studies, University of Alabama, Tuscaloosa, AL 35487, USA \\ ${ }^{2}$ Department of Finance, Insurance and Real Estate, Laval University, Quebec City, Quebec G1V oA6, Canada, E-mail: \\ richard.luger@fsa.ulaval.ca
}

\begin{abstract}
:
We extend the class of linear quantile autoregression models by allowing for the possibility of Markov-switching regimes in the conditional distribution of the response variable. We also develop a Gibbs sampling approach for posterior inference by using data augmentation and a location-scale mixture representation of the asymmetric Laplace distribution. Bayesian calculations are easily implemented, because all complete conditional densities used in the Gibbs sampler have closed-form expressions. The proposed Gibbs sampler provides the basis for a stepwise re-estimation procedure that ensures non-crossing quantiles. Monte Carlo experiments and an empirical application to the U.S. real interest rate show that both inference and forecasting are improved when the quantile monotonicity restriction is taken into account.
\end{abstract}

Keywords: asymmetric Laplace distribution, Gibbs sampler, non-crossing quantiles, quantile regression, regime changes

JEL classification: C11, C22, C51

DOI: $10.1515 /$ snde-2016-0078

\section{Introduction}

The class of linear quantile autoregression (QAR) models of Koenker and Xiao (2006) has proven to be particularly useful for studying asymmetric dynamics and local persistence in many economic and financial time series. Examples include real estate prices (Galvao, Montes-Rojas \& Park, 2013; Lee, Lee \& Lee, 2014), stock prices and exchange rates (Ferreira, 2011; Baur, Dimpfl \& Jung, 2012; Yang, Tu \& Zeng, 2014), and more general business cycle and inflation indicators (Manzan, 2015). Our objective in this paper is to extend the class of QAR models by allowing for the possibility of Markov-switching regimes that would influence the conditional quantiles. Indeed, the presence of regime changes in the conditional distribution of the response variable would obviously affect its quantiles. The importance of allowing for such effects can also be recognized from the work of $\mathrm{Qu}(2008)$ who proposes testing procedures for structural change in conditional quantiles.

Since the seminal contribution of Hamilton (1989), Markov-switching specifications have become an immensely popular approach to model structural changes in the behaviour of economic and financial time series; see Piger (2009) for an overview. A distinctive feature of the Markov-switching approach is that the regime changes are endogenous to the model, as opposed to being treated exogenously like in the classic approach to structural changes by Chow (1960) which assumes a priori knowledge about how to classify the data between regimes. Building on the earlier work of Goldfeld and Quandt (1973), the Hamilton (1989) approach specifies the regimes as being determined by a discrete-time, discrete-state Markov chain with unknown transition probabilities. This process is assumed to be recurrent, meaning that it can move from one state to any other state at any time. As in the well-known Kalman filter, the unobserved state can be inferred from the observable time series and the sample likelihood function can then be recursively computed. Our specification also complements the related extension of the QAR model proposed by Galvao, Montes-Rojas, and Olmo (2011) in which regime changes are triggered when the time series passes (quantile-specific) threshold values, like in the self-exciting threshold autoregression models of Tong (1983).

Following Albert and Chib (1993), McCulloch and Tsay (1994), Chib (1996), and Frühwirth-Schnatter (2001), we adopt a Bayesian approach to inference based on data augmentation such that the latent states can be analyzed along with the other unknown model parameters through Gibbs sampling. The advantage of the Gibbs sampling approach to the analysis of Markov-switching models has long been recognized. For example, Albert and Chib (1993) remark that such an approach avoids the direct calculation of the likelihood function needed for maximum likelihood estimation. Moreover, the posterior distributions of all unknown parameters (and 
functions thereof) are fully tractable and easy to simulate from. By treating the unobserved states as missing data, this approach also provides posterior distributions of the states, integrated (by marginalization) over all the other model parameters.

The Bayesian analysis of quantile regression models rests on the connection between the quantile estimation problem and the likelihood function under an asymmetric Laplace distribution, established by Yu and Moyeed (2001) and Tsionas (2003). It is important to note, however, that as in Koenker and Machado (1999) the asymmetric Laplace distribution is not assumed for the data generating process, but is merely used to obtain a quasi-likelihood function. Other examples of such a parametrization for the purpose Bayesian inference include Geraci and Bottai (2007), Kottas and Krnjajić (2009), Yue and Rue (2011), Gerlach, Chen, and Chan (2011), and Chen et al. (2012).

The asymmetric Laplace distribution can be expressed as a location-scale mixture of exponential and normal distributions (Kotz, Kozubowski \& Podgórski, 2001). Following Kozumi and Kobayashi (2011), we exploit this representation to develop a Gibbs sampling approach wherein all complete conditional densities have closedform expressions. An estimate of the marginal likelihood can then be calculated from the Gibbs output using the method of Chib (1995). The marginal likelihood is the key ingredient for model selection and discrimination in Bayesian statistics; see the discussion in Kass and Raftery (1995).

We use the Gibbs sampler to solve the well-known quantile crossing problem that may arise when quantile models are fitted separately for each considered quantile probability level, $\tau$. This common practice of treating the quantile levels independently of one another can yield fitted quantile curves that cross one another, thereby leading to a nonsensical response distribution. Indeed, quantile monotonicity requires the quantiles to be increasing as a function of $\tau$, meaning that any well-defined distribution must necessarily have non-crossing quantiles. Koenker and Xiao (2006), $\$ 4$ remark that the crossing problem appears more acute in QAR models than in ordinary quantile regressions with exogenous covariates, since the support of the regressors is determined within the autoregressive model.

This quantile crossing problem is potentially worse for non-linear quantile autoregression models, like the threshold specification of Galvao, Montes-Rojas, and Olmo (2011) and the Markov-switching specification developed here. Fortunately, the Gibbs sampler provides the basis for a stepwise re-estimation procedure that ensures non-crossing quantiles. As in Gelfand, Smith, and Lee (1992), draws from the constrained posterior distribution are obtained straightforwardly by retaining the Gibbs draws that satisfy the non-crossing condition when sampling the unconstrained posterior distribution. As far as we know, this is the only way to carry out full Bayesian calculations while avoiding well-nigh impossible numerical integrations over high-dimensional sets defined by complex restrictions involving the model parameters and the data.

It is important to note that the proposal in a related paper by Ye et al. (2016) is to allow for Markov-switching parameters in an ordinary quantile regression with exogenous (independent) regressors, not a quantile autoregression. This is a fundamental difference with what we propose here. Indeed, as we already mentioned, QAR models are more likely to suffer from the quantile crossing problem than ordinary quantile regressions since the regressors are endogenous to the model. Ye et al. (2016) also exploit the asymmetric Laplace connection to devise a (quasi) maximum likelihood estimation (MLE) approach for point estimation, but they do not establish any distributional theory to guide inference. Moreover, their MLE-based approach treats each quantile level separately, which means that it may yield crossing quantiles even though their model is less prone to this problem. In sharp contrast to Ye et al. (2016), our Gibbs sampling approach offers a complete Bayesian methodology not only for estimation, but also inference, model selection, and ensuring non-crossing quantiles in quantile regresison models. It is also important to note that our complete closed-form Gibbs sampling approach can be applied in any quantile regression model with endogenous or exogenous covariates, and whether Markovswitching effects are allowed for or not. In our empirical application, for instance, we estimate non-crossing quantiles with the linear QAR model as well as the Markov-switching QAR model.

The current paper is organized as follows. Section 2 begins by introducing the QAR models of Koenker and Xiao (2006), then shows the asymmetric Laplace connection, and describes the proposed Markov-switching quantile autoregression models. Section 3 develops the Gibbs sampling algorithm based on a location-scale mixture representation of the asymmetric Laplace distribution. There are two variants of the approach, depending on how the state variables are sampled (single- or multi-move Gibbs sampling). Section 4 explains the computation of the marginal likelihood used for model comparisons and the stepwise re-estimation procedure to ensure non-crossing quantiles. Section 5 presents some simulation evidence about the relative performance of the Gibbs samplers in the quantile regression context. Section 6 presents the empirical application to the U.S. real interest rate, which illustrates the gains obtained by enforcing the quantile monotonicity restriction. Section 7 concludes and the computational details are given in the appendices. 


\section{Markov-switching quantile autoregression}

Suppose we have a response variable of interest $y_{t}$ whose time- $t$ conditional quantiles we wish to model as a function of past information. The linear quantile autoregression (QAR) model of Koenker and Xiao (2006) specifies the $\tau$ th conditional quantile of $y_{t}$ as

$$
Q_{y_{t}}\left(\tau \mid \mathbf{y}_{t-p: t-1}\right)=c(\tau)+\sum_{j=1}^{p} \phi_{j}(\tau) y_{t-j}
$$

where $\mathbf{y}_{t_{1}: t_{2}}$ refers to observations $y_{t_{1}}, \ldots, y_{t_{2}}$, for $t_{1} \leq t_{2}$, with the convention that $\mathbf{y}=\mathbf{y}_{1: T}$. Given a specified value of the quantile level $\tau \in(0,1)$, the parameters of the QAR $(p)$ model in (1) can be estimated by solving the following minimization problem:

$$
\min \sum_{t=p+1}^{T} \rho_{\tau}\left(y_{t}-Q_{y_{t}}\left(\tau \mid \mathbf{y}_{t-p: t-1}\right)\right),
$$

by choice of $c(\tau), \phi_{1}(\tau), \ldots, \phi_{p}(\tau)$, and where $\rho_{\tau}(\cdot)$ is the asymmetric absolute deviation loss function defined as $\rho_{\tau}(u)=u(\tau-\llbracket[u<0])$ (Koenker \& Bassett, 1978). Here $\mathbb{\square}[A]$ is the indicator function which equals one when event $A$ is true, and zero otherwise. For the median $\tau=0.5$, the loss function in (2) becomes $\rho_{\tau}(u)=0.5|u|$.

Koenker and Machado (1999) explain that the parameters of linear quantile models can also be estimated by (quasi) maximum likelihood. To see how, consider the QAR $(p)$ model specified in parametric distributional form as

$$
y_{t}=c(\tau)+\sum_{j=1}^{p} \phi_{j}(\tau) y_{t-j}+\delta \varepsilon_{t}
$$

where $\delta>0$ is a scale parameter and $\left\{\varepsilon_{t}\right\}$ are i.i.d. according to the asymmetric Laplace distribution with probability density function

$$
f\left(\varepsilon_{t}\right)=\tau(1-\tau) \exp \left(-\rho_{\tau}\left(\varepsilon_{t}\right)\right) .
$$

The conditional density function of $y_{t}$ for a given $\tau$ then becomes

$$
f\left(y_{t} \mid \mathbf{y}_{t-p: t-1}\right)=\frac{\tau(1-\tau)}{\delta} \exp \left\{-\rho_{\tau}\left(\frac{y_{t}-Q_{y_{t}}\left(\tau \mid \mathbf{y}_{t-p: t-1}\right)}{\delta}\right)\right\}
$$

and, conditional on $\mathbf{y}_{1: p}$, the sample likelihood can be written as

$$
\mathscr{L}\left(c(\tau), \phi_{1}(\tau), \ldots, \phi_{p}(\tau), \delta\right)=\frac{\tau^{T-p}(1-\tau)^{T-p}}{\delta^{T-p}} \exp \left\{-\delta^{-1} \sum_{t=p+1}^{T} \rho_{\tau}\left(y_{t}-Q_{y_{t}}\left(\tau \mid \mathbf{y}_{t-p: t-1}\right)\right)\right\} .
$$

Since the negative of the loss function appears in the exponent of this expression, maximization of (5) is equivalent to solving the minimization problem in (2). As Yu and Moyeed (2001) and Tsionas (2003) explain, the asymmetric Laplace distribution provides a natural pathway for the Bayesian analysis of quantile regression models. Note also that the value of $\delta$ does not matter for the estimation of the correct quantiles by maximizing (5). But rather than fixing it to a constant value, using $\delta$ as a free scale parameter clearly makes the assumed asymmetric Laplace distribution more flexible to capture the true (unknown) error distribution. Other examples of such a parametrization for Bayesian inference include Geraci and Bottai (2007), Kottas and Krnjajić (2009), Yue and Rue (2011), Gerlach, Chen, and Chan (2011), and Chen et al. (2012). to

The starting point for the developed model is the observation that the QAR $(p)$ model in form (3) is equivalent

$$
\begin{aligned}
& y_{t}=\mu(\tau)+\eta_{t} \\
& \eta_{t}=\sum_{j=1}^{p} \phi_{j}(\tau) \eta_{t-j}+\delta \varepsilon_{t}
\end{aligned}
$$


where $\left\{\varepsilon_{t}\right\}$ are i.i.d. according to the asymmetric Laplace distribution with density (4) so that $\mu(\tau)$ is the location of the $\tau$ th conditional quantile in this model with autocorrelated errors. Indeed, since $\eta_{t}=y_{t}-\mu(\tau)$, for all $t,(6)$ can be rewritten as (3) with $c(\tau)=\mu(\tau)\left(1-\sum_{j=1}^{p} \phi_{j}(\tau)\right)$. The presence of regime changes in the true conditional distribution of $y_{t}$ would obviously affect the location of its quantiles. Following Hamilton (1989), the quantile model in (6) can be generalized to account for the possible presence of such regimes as follows:

$$
\begin{aligned}
y_{t} & =\mu\left(\tau, s_{t}\right)+\eta_{t}, \\
\mu\left(\tau, s_{t}\right) & =\sum_{i=1}^{K} \mu_{i}(\tau) \square\left[s_{t}=i\right], \\
\eta_{t} & =\sum_{j=1}^{p} \phi_{j}(\tau) \eta_{t-j}+\delta \varepsilon_{t}
\end{aligned}
$$

where $s_{t}$ is a latent variable taking values in the set $\{1, \ldots, K\}$ according to a Markov chain with one-step transition probability matrix $\mathbf{P}$ whose elements are defined as

$$
\begin{aligned}
p_{i j} & =\operatorname{Pr}\left(s_{t}=j \mid s_{t-1}=i\right), \\
\sum_{j=1}^{K} p_{i j} & =1, \text { for all } i \in\{1, \ldots, K\} .
\end{aligned}
$$

The term $\mu\left(\tau, s_{t}\right)$ in (7) is thus the location of the $\tau$ th conditional quantile of $y_{t}$ given the past of $y_{t}$ itself and the current regime $s_{t}$. The proposed Markov-switching quantile autoregression (MSQAR) model can then be rewritten as

$$
y_{t}=\mu\left(\tau, s_{t}\right)+\sum_{j=1}^{p} \phi_{j}(\tau)\left(y_{t-j}-\mu\left(\tau, s_{t-j}\right)\right)+\delta \varepsilon_{t}
$$

since $\eta_{t}=y_{t}-\mu\left(\tau, s_{t}\right)$, for all $t$, in (7). For a specified $\tau$, the conditional density function of $y_{t}$ given $y_{t-1}, \ldots, y_{t-p}$ and $s_{t}, \ldots, s_{t-p}$ becomes

$$
f\left(y_{t} \mid \mathbf{y}_{t-p: t-1}, \mathbf{s}_{t-p: t}\right)=\frac{\tau(1-\tau)}{\delta} \exp \left\{-\rho_{\tau}\left(\frac{y_{t}-Q_{y_{t}}\left(\tau \mid \mathbf{y}_{t-p: t-1}, \mathbf{s}_{t-p: t}\right)}{\delta}\right)\right\},
$$

where the $\tau$ th conditional quantile function is given by

$$
Q_{y_{t}}\left(\tau \mid \mathbf{y}_{t-p: t-1}, \mathbf{s}_{t-p: t}\right)=\mu\left(\tau, s_{t}\right)+\sum_{j=1}^{p} \phi_{j}(\tau)\left(y_{t-j}-\mu\left(\tau, s_{t-j}\right)\right)
$$

Here $\mathbf{s}_{t_{1}: t_{2}}$ refers to $s_{t_{1}}, \ldots, s_{t_{2}}$, for $t_{1} \leq t_{2}$, and we let $\mathbf{s}=\mathbf{s}_{1: T}$. Observe that it would not make sense to allow for switching in $\delta$, since the quantile regimes cannot be identified through this reduced-form parameter. Note also that if $\mu_{i}(\tau)=\mu_{j}(\tau)$ for all $i, j$, then the $K$-regime $\operatorname{MSQAR}(K, p)$ model in $(11)$ collapses to the linear $(K=1$ regime) $\operatorname{QAR}(p)$ model in (1). In this case we have $\mu(\tau)=c(\tau) /\left(1-\sum_{j=1}^{p} \phi_{j}(\tau)\right)$, assuming of course that there are no unit roots so the denominator is different from zero.

The MSQAR model in form (9) can be expressed more compactly as

$$
\phi(\tau, L)\left(y_{t}-\mu\left(\tau, s_{t}\right)\right)=\delta \varepsilon_{t}
$$

where $\phi(\tau, L)=\left(1-\phi_{1}(\tau) L-\cdots-\phi_{p}(\tau) L^{p}\right)$ is a $p$ th order polynomial in the lag operator $L$, defined such that $L^{k} z_{t}=z_{t-k}$ for $k \geq 0$. The following assumptions are made:

1. $\mu_{1}(\tau)<\mu_{2}(\tau)<\cdots<\mu_{K}(\tau)$.

2. All the roots of $\phi(\tau, L)=0$ lie outside the unit circle.

3. $p_{i j}>0$ for all $i, j \in\{1, \ldots, K\}$. 
4. $\operatorname{Pr}\left(s_{1}=i\right)=1 / K$ for all $i \in\{1, \ldots, K\}$.

The first three assumptions are standard for Markov-switching time-series models. Assumption 1 ensures the identification of the regimes, while Assumption 2 imposes stationarity given the sequence of state variables. Assumption 3 guarantees that the Markov chain is irreducible, i.e. starting $s_{t}$ from an arbitrary state $i \in\{1, \ldots$, $K\}$, any state $j \in\{1, \ldots, K\}$ is reachable in finite time. This assumption is also needed for identification purposes because if a state is never visited then the associated parameters cannot be identified. Assumption 4 treats the initial state as a random draw from a uniform distribution, independently of the transition probabilities; see Frühwirth-Schnatter (2006), Ch. 10 for a discussion of the computational advantages of this assumption.

Under these assumptions, the joint density of $\mathbf{y}_{p+1: T}$ and $\mathbf{s}$, conditional on $\mathbf{y}_{1: p}$, is

$$
f\left(\mathbf{y}_{p+1: T}, \mathbf{s} \mid \mathbf{y}_{1: p}\right)=f\left(\mathbf{y}_{p+1: T} \mid \mathbf{y}_{1: p}, \mathbf{s}\right) \times p(\mathbf{s} \mid \mathbf{p}),
$$

which does not constitute the likelihood function. Indeed, the likelihood function for $\mathbf{y}_{p+1: T}$ is obtained by integrating out the state variables. Appendix A presents an algorithm to compute the MSQAR likelihood function. ${ }^{1}$

\section{Gibbs sampling}

The asymmetric Laplace distribution admits various mixture representations (Kotz, Kozubowski \& Podgórski, 2001). Following Kozumi and Kobayashi (2011), the Gibbs sampling algorithm developed here uses a representation based on a mixture of exponential and normal distributions. Specifically, if $\varepsilon_{t}$ follows the asymmetric Laplace distribution with density (4), then $\varepsilon_{t}$ can be represented as

$$
\varepsilon_{t}=\gamma e_{t}+\xi \sqrt{e_{t}} z_{t}
$$

with

$$
\gamma=\frac{1-2 \tau}{\tau(1-\tau)} \text { and } \xi^{2}=\frac{2}{\tau(1-\tau)}
$$

where $e_{t} \sim \mathscr{E}(1)$, a standard exponential distribution, and $z_{t} \sim N(0,1)$, independently of $e_{t}$. With this mixture representation, model (9) can be equivalently rewritten as

$$
y_{t}=\mu\left(\tau, s_{t}\right)+\sum_{j=1}^{p} \phi_{j}(\tau)\left(y_{t-j}-\mu\left(\tau, s_{t-j}\right)\right)+\delta \gamma e_{t}+\delta \xi \sqrt{e_{t}} z_{t} .
$$

However, as Kozumi and Kobayashi (2011) explain, such an expression is not convenient for Gibbs sampling because the scale parameter $\delta$ appears in the conditional location of $y_{t}$. This issue is resolved by working instead with the reparameterization:

$$
y_{t}=\mu\left(\tau, s_{t}\right)+\sum_{j=1}^{p} \phi_{j}(\tau)\left(y_{t-j}-\mu\left(\tau, s_{t-j}\right)\right)+\gamma v_{t}+\xi \sqrt{\delta v_{t}} z_{t},
$$

where $v_{t}=\delta e_{t}$ and we let $\mathbf{v}=\mathbf{v}_{p+1: T}=\left(v_{p+1}, \ldots, v_{T}\right)$.

The model parameters are collected in $\boldsymbol{\theta}(\tau)=\left(\boldsymbol{\mu}(\tau)^{\prime}, \boldsymbol{\phi}(\tau)^{\prime}, \delta, \mathbf{p}^{\prime}\right)^{\prime}$, where $\boldsymbol{\mu}(\tau)=\left(\mu_{1}(\tau), \ldots, \mu_{K}(\tau)\right)^{\prime}$, $\phi(\tau)=\left(\phi_{1}(\tau), \ldots, \phi_{p}(\tau)\right)^{\prime}$, and $\mathbf{p}=\operatorname{vec}(\mathbf{P})$ stacks the elements of the transition probability matrix into a column vector. The Gibbs sampler is an iterative procedure that can be started using any set of values in the support of the posterior distribution $\pi(\boldsymbol{\theta}(\tau) \mid \mathbf{y})$ and will produce a Markov chain $\left\{\boldsymbol{\theta}(\tau)^{n}\right\}_{n=1}^{N}$ with equilibrium distribution equal to this target posterior distribution; see Casella and George (1992) for an introduction and Tierney (1994) for a more detailed treatment. Note that $\mathbf{v}$ and $\mathbf{s}$ are latent, so data augmentation will be used when sampling from the posterior distribution. Specifically, given a previous Gibbs draw $\left(\mathbf{s}^{n-1}, \mathbf{p}^{n-1}, \boldsymbol{\mu}(\tau)^{n-1}, \boldsymbol{\phi}(\tau)^{n-1}, \mathbf{v}^{n-1}, \delta^{n-1}\right)$, the next one is obtained by sampling iteratively from the following full conditional distributions: 


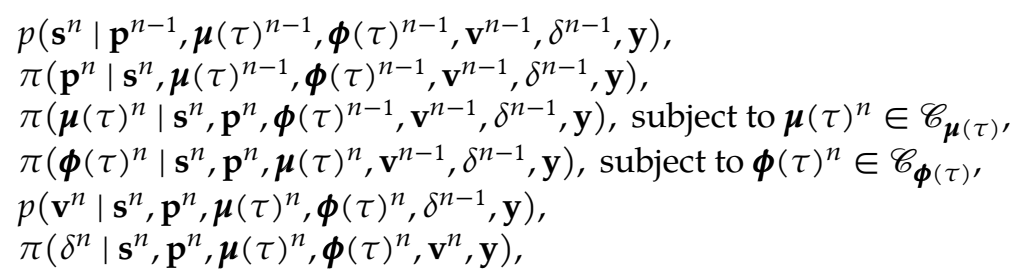

where $\mathscr{C}_{\boldsymbol{\mu}(\tau)}=\left\{\boldsymbol{\mu}(\tau): \mu_{1}(\tau)<\mu_{2}(\tau)<\cdots<\mu_{K}(\tau)\right\}$ and $\mathscr{C}_{\boldsymbol{\phi}(\tau)}=\{\boldsymbol{\phi}(\tau)$ : all the roots of $\phi(\tau, L)=$ 0 lie outside the unit circle\} are the constraint sets for $\boldsymbol{\mu}(\tau)$ and $\boldsymbol{\phi}(\tau)$ under Assumption 1 and Assumption 2 , respectively. These steps are repeated a large number of times until the Markov chain has achieved convergence. After the burn-in iterations, each complete pass through the Gibbs steps yields a draw from the joint posterior density $\pi(\mathbf{s}, \mathbf{p}, \boldsymbol{\mu}(\tau), \boldsymbol{\phi}(\tau), \mathbf{v}, \delta \mid \mathbf{y})$. These draws from the sampling algorithm are denoted by $\left\{\mathbf{s}^{n}, \mathbf{p}^{n}, \boldsymbol{\mu}(\tau)^{n}, \boldsymbol{\phi}(\tau)^{n}, \mathbf{v}^{n}, \delta^{n} \mid \mathbf{y}\right\}_{n=1}^{N}$. Appendix B explains in detail how to generate draws from each of the conditional distributions making up the steps of the Gibbs sampling procedure.

\section{Specification issues}

The proposed MSQAR model applies to a specified quantile probability level $\tau$. A typical quantile regression analysis, however, might involve several probability levels $\tau_{1}<\cdots<\tau_{q}$. Two issues arise when several quantile probability levels $\tau$ are considered. The first is that the models at the considered quantile levels each yield an inference about the latent Markov chain. This begs the question: which one should be retained?

The second issue is that the fitted quantiles can cross one another, since the models (defined for each $\tau$ ) are fitted separately. In other words, if the models are estimated separately for each of the $q$ desired probability levels, then the resulting conditional quantile functions may not be monotonically increasing in $\tau$. This is the well-known quantile crossing problem, which obviously leads to a nonsensical response distribution since any distribution must necessarily have non-crossing quantiles in order to be well defined. We solve this problem by proposing a stepwise procedure similar to Wu and Liu (2009), whereby the quantiles are refitted sequentially while constraining the current curve not to cross the previous one.

\subsection{Posterior state classification}

Our proposal is to first estimate an MSQAR for each quantile level $\tau_{1}, \ldots, \tau_{q}$ separately and to compute the log marginal likelihood estimates $\log \hat{\pi}\left(\mathbf{y} \mid \tau_{i}\right), i=1, \ldots, q$, corresponding to each of these models. The marginal likelihood is related to the prior, posterior, and sample density functions via the equality

$$
\pi\left(\mathbf{y} \mid \tau_{i}\right)=\frac{f\left(\mathbf{y} \mid \boldsymbol{\theta}\left(\tau_{i}\right)\right) \pi\left(\boldsymbol{\theta}\left(\tau_{i}\right)\right)}{\pi\left(\boldsymbol{\theta}\left(\tau_{i}\right) \mid \mathbf{y}\right)},
$$

which holds at all admissible points of the parameter space. So for given values $\theta\left(\tau_{i}\right)^{*}$ of the model parameters, we can obtain an estimate of $\log \pi\left(\mathbf{y} \mid \tau_{i}\right)$ using

$$
\log \hat{\pi}\left(\mathbf{y} \mid \tau_{i}\right)=\log f\left(\mathbf{y} \mid \boldsymbol{\theta}\left(\tau_{i}\right)^{*}\right)+\log \pi\left(\boldsymbol{\theta}\left(\tau_{i}\right)^{*}\right)-\log \hat{\pi}\left(\boldsymbol{\theta}\left(\tau_{i}\right)^{*} \mid \mathbf{y}\right),
$$

where $\hat{\pi}\left(\boldsymbol{\theta}\left(\tau_{i}\right)^{*} \mid \mathbf{y}\right)$ is an estimate of the posterior ordinate at the chosen parameter values. In principle, $\boldsymbol{\theta}\left(\tau_{i}\right)^{*}$ could be any point in the space of admissible values. ${ }^{2}$ Here we follow Chib (1995) and use the posterior mean; see Appendix $C$ for the detailed computation of (13).

Let $\tau_{i^{*}}$ refer to the model achieving the highest marginal likelihood among the quantile levels $\tau_{1}<\cdots<$ $\tau_{i^{*}-1}<\tau_{i^{*}}<\tau_{i^{*}+1}<\cdots<\tau_{q}$. This model is used to obtain a classification of regimes at each time period from the output of the Gibbs sampler, written here in expanded form as $\left\{s_{1}^{n}, \ldots, s_{T^{\prime}}^{n}, \mathbf{p}^{n}, \boldsymbol{\mu}\left(\tau_{i^{*}}\right)^{n}, \boldsymbol{\phi}\left(\tau_{i^{*}}\right)^{n}, \mathbf{v}^{n}, \delta^{n}\right\}_{n=1}^{N}$ with $N$ being the number of draws from the posterior density. Following Bauwens, Preminger, and Rombouts (2010) and Billio, Casarin, and Osuntuyi (2014), we identify $\hat{s}_{t}$ as the time- $t$ state with the highest posterior probability. Specifically, we estimate the posterior state probabilities as

$$
\operatorname{Pr}\left(s_{t}=i \mid \mathbf{y}\right)=N^{-1} \sum_{i=1}^{N} \square\left[s_{t}^{n}=i\right],
$$


for $t=1, \ldots, T$ and $i=1, \ldots, K$. Note that these posterior state probabilities may be considered as "smoothed" probabilities, since they are based on the full sample, $\mathbf{y}$ (Kim \& Nelson, 1999, p. 233). The associated sequence of fitted states is denoted $\hat{\mathbf{s}}=\left\{\hat{s}_{t}\right\}_{t=1}^{T}$, which results from applying the classification rule: set $\hat{s}_{t}=i$ if $\operatorname{Pr}\left(s_{t}=i \mid \mathbf{y}\right)$ is the maximal value among $\operatorname{Pr}\left(s_{t}=1 \mid \mathbf{y}\right), \ldots, \operatorname{Pr}\left(s_{t}=K \mid \mathbf{y}\right) .^{3}$

In many practical applications, it may be convenient to simply set $\tau_{i^{*}}=0.5$. This would be a reasonable choice if the true error distribution is unimodal and symmetric around zero like a centered normal distribution (cf. Wu \& Liu, 2009) and this choice also yields computational savings.

\subsection{Non-crossing quantiles}

The next step consists of sequentially re-estimating the conditional quantile functions subject to the noncrossing restrictions. Under these constraints the parameter vector $\boldsymbol{\theta}(\tau)$ is restricted to lie in a set $\Xi_{\mathrm{y}, \boldsymbol{\tau}}$ which ensures that the conditional quantiles at level $\tau$ do not cross those at any other level. The $\tau$ th conditional quantiles that we wish to restrict are

$$
Q_{y_{t}}(\tau \mid \boldsymbol{\mu}(\tau), \boldsymbol{\phi}(\tau), \mathbf{y}, \hat{\mathbf{s}})=\mu\left(\tau, \hat{s}_{t}\right)+\sum_{j=1}^{p} \phi_{j}(\tau)\left(y_{t-j}-\mu\left(\tau, \hat{s}_{t-j}\right)\right), \quad t=1, \ldots, T,
$$

where $\hat{\mathbf{s}}$ is the sequence of fitted states inferred from the model at level $\tau_{i^{*}}$. Starting with the level $\tau_{i^{*}}$ at which the quantiles are unconstrained, the subsequent constraint sets $\Xi_{\mathbf{y}, \tau_{j-1}}$, for $j=i^{*}, i^{*}-1, \ldots, 2$, are then determined by the inequality restrictions:

$$
Q_{y_{t}}\left(\tau_{j-1} \mid \boldsymbol{\mu}\left(\tau_{j-1}\right), \boldsymbol{\phi}\left(\tau_{j-1}\right), \mathbf{y}, \hat{\mathbf{s}}\right) \leq Q_{y_{t}}\left(\tau_{j} \mid \hat{\boldsymbol{\mu}}\left(\tau_{j}\right), \hat{\boldsymbol{\phi}}\left(\tau_{j}\right), \mathbf{y}, \hat{\mathbf{s}}\right), \quad t=1, \ldots, T,
$$

and $\Xi_{\mathrm{y}, \tau_{j+1}}$, for $j=i^{*}, i^{*}+1, \ldots, q-1$, are determined by

$$
Q_{y_{t}}\left(\tau_{j+1} \mid \boldsymbol{\mu}\left(\tau_{j+1}\right), \boldsymbol{\phi}\left(\tau_{j+1}\right), \mathbf{y}, \hat{\mathbf{s}}\right) \geq Q_{y_{t}}\left(\tau_{j} \mid \hat{\boldsymbol{\mu}}\left(\tau_{j}\right), \hat{\boldsymbol{\phi}}\left(\tau_{j}\right), \mathbf{y}, \hat{\mathbf{s}}\right), \quad t=1, \ldots, T,
$$

where $\hat{\mu}\left(\tau_{j}\right)$ and $\hat{\phi}\left(\tau_{j}\right)$ refer to the posterior mean estimates at the previous level $\tau_{j}$. Observe that once the constraint sets $\Xi_{\mathbf{y}, \tau_{j}}, j=1, \ldots, q$, are determined, the constrained Bayesian models (likelihood $\times$ prior) are given by

$$
\begin{cases}f\left(\mathbf{y} \mid \boldsymbol{\theta}\left(\tau_{j}\right)\right) \pi\left(\boldsymbol{\theta}\left(\tau_{j}\right)\right), & \left(\mathbf{y}, \boldsymbol{\theta}\left(\tau_{j}\right)\right) \in \Xi_{\tau_{j^{\prime}}} \\ 0, & \left(\mathbf{y}, \boldsymbol{\theta}\left(\tau_{j}\right)\right) \notin \Xi_{\tau_{j^{\prime}}}\end{cases}
$$

where $\Xi_{\tau_{j}}=\left\{\left(\mathbf{y}, \boldsymbol{\theta}\left(\tau_{j}\right)\right): \boldsymbol{\theta}\left(\tau_{j}\right) \in \Xi_{y, \tau_{j}}\right\}$. The posterior distribution for $\boldsymbol{\theta}\left(\tau_{j}\right)$, given the constraints, is then simply the unconstrained posterior appropriately normalized so that

$$
\pi\left(\boldsymbol{\theta}\left(\tau_{j}\right) \mid \mathbf{y}\right)=\frac{f\left(\mathbf{y} \mid \boldsymbol{\theta}\left(\tau_{j}\right)\right) \pi\left(\boldsymbol{\theta}\left(\tau_{j}\right)\right)}{\int_{\Xi_{y, \tau_{j}}} f\left(\mathbf{y} \mid \boldsymbol{\theta}\left(\tau_{j}\right)\right) \pi\left(\boldsymbol{\theta}\left(\tau_{j}\right)\right)}, \quad \boldsymbol{\theta}\left(\tau_{j}\right) \in \Xi_{y, \tau_{j}} .
$$

It is important to realize that a direct evaluation of this expression is infeasible as it involves numerical integrations over $\Xi_{y, \tau_{j}}$, which is a high-dimensional set defined by very complex restrictions involving the model parameters and the data. And there are $q-1$ such expressions, defined for $j=1, \ldots, q$, save for $j=i^{*}$.

Fortunately, it is straightforward to obtain a constrained posterior distribution with the Gibbs sampler. Regardless of how complicated a constraint set is, Gelfand, Smith, and Lee (1992) show that the Gibbs sampler can be implemented by identifying the full conditionals under the unconstrained model and then restricting the cross-section. This can be done simply by generating from the unconstrained full conditional and retaining the variate value only if it falls in the cross-section constraint region; see Gelfand, Smith, and Lee (1992) for more details and other examples. In our context, this approach consists of sampling iteratively from $\pi\left(\boldsymbol{\mu}\left(\tau_{j-1}\right)^{n} \mid \boldsymbol{\phi}\left(\tau_{j-1}\right)^{n-1}, \mathbf{v}^{n-1}, \delta^{n-1}, \mathbf{y}, \widehat{\mathbf{s}}\right)$, subject to

$$
Q_{y_{t}}\left(\tau_{j-1} \mid \boldsymbol{\mu}\left(\tau_{j-1}\right)^{n}, \boldsymbol{\phi}\left(\tau_{j-1}\right)^{n-1}, \mathbf{y}, \hat{\mathbf{s}}\right) \leq Q_{y_{t}}\left(\tau_{j} \mid \hat{\boldsymbol{\mu}}\left(\tau_{j}\right), \hat{\boldsymbol{\phi}}\left(\tau_{j}\right), \mathbf{y}, \widehat{\mathbf{s}}\right), \quad t=1, \ldots, T,
$$


$\pi\left(\boldsymbol{\phi}\left(\tau_{j-1}\right)^{n} \mid \boldsymbol{\mu}\left(\tau_{j-1}\right)^{n}, \mathbf{v}^{n-1}, \delta^{n-1}, \mathbf{y}, \hat{\mathbf{s}}\right)$, subject to

$$
Q_{y_{t}}\left(\tau_{j-1} \mid \boldsymbol{\mu}\left(\tau_{j-1}\right)^{n}, \boldsymbol{\phi}\left(\tau_{j-1}\right)^{n}, \mathbf{y}, \hat{\mathbf{s}}\right) \leq Q_{y_{t}}\left(\tau_{j} \mid \hat{\boldsymbol{\mu}}\left(\tau_{j}\right), \hat{\boldsymbol{\phi}}\left(\tau_{j}\right), \mathbf{y}, \hat{\mathbf{s}}\right), \quad t=1, \ldots, T,
$$

$\pi\left(\mathbf{v}^{n} \mid \boldsymbol{\mu}\left(\tau_{j-1}\right)^{n}, \boldsymbol{\phi}\left(\tau_{j-1}\right)^{n}, \delta^{n-1}, \mathbf{y}, \hat{\mathbf{s}}\right)$, $\pi\left(\delta^{n} \mid \hat{\mathbf{s}}, \boldsymbol{\mu}\left(\tau_{j-1}\right)^{n}, \boldsymbol{\phi}\left(\tau_{j-1}\right)^{n}, \mathbf{v}^{n}, \mathbf{y}\right)$,

for $j=i^{*}, i^{*}-1, \ldots, 2$, and then from

$\pi\left(\boldsymbol{\mu}\left(\tau_{j+1}\right)^{n} \mid \boldsymbol{\phi}\left(\tau_{j+1}\right)^{n-1}, \mathbf{v}^{n-1}, \delta^{n-1}, \mathbf{y}, \widehat{\mathbf{s}}\right)$, subject to

$$
Q_{y_{t}}\left(\tau_{j+1} \mid \boldsymbol{\mu}\left(\tau_{j+1}\right)^{n}, \boldsymbol{\phi}\left(\tau_{j+1}\right)^{n-1}, \mathbf{y}, \hat{\mathbf{s}}\right) \geq Q_{y_{t}}\left(\tau_{j} \mid \hat{\boldsymbol{\mu}}\left(\tau_{j}\right), \hat{\boldsymbol{\phi}}\left(\tau_{j}\right), \mathbf{y}, \widehat{\mathbf{s}}\right), \quad t=1, \ldots, T,
$$

$\pi\left(\phi\left(\tau_{j+1}\right)^{n} \mid \boldsymbol{\mu}\left(\tau_{j+1}\right)^{n}, \mathbf{v}^{n-1}, \delta^{n-1}, \mathbf{y}, \hat{\mathbf{s}}\right)$, subject to

$$
Q_{y_{t}}\left(\tau_{j+1} \mid \boldsymbol{\mu}\left(\tau_{j+1}\right)^{n}, \boldsymbol{\phi}\left(\tau_{j+1}\right)^{n}, \mathbf{y}, \hat{\mathbf{s}}\right) \geq Q_{y_{t}}\left(\tau_{j} \mid \hat{\boldsymbol{\mu}}\left(\tau_{j}\right), \hat{\boldsymbol{\phi}}\left(\tau_{j}\right), \mathbf{y}, \hat{\mathbf{s}}\right), \quad t=1, \ldots, T,
$$

$\pi\left(\mathbf{v}^{n} \mid \boldsymbol{\mu}\left(\tau_{j+1}\right)^{n}, \boldsymbol{\phi}\left(\tau_{j+1}\right)^{n}, \delta^{n-1}, \mathbf{y}, \widehat{\mathbf{s}}\right)$,

$\pi\left(\delta^{n} \mid \hat{\mathbf{s}}, \boldsymbol{\mu}\left(\tau_{j+1}\right)^{n}, \boldsymbol{\phi}\left(\tau_{j+1}\right)^{n}, \mathbf{v}^{n}, \mathbf{y}\right)$,

for $j=i^{*}, i^{*}+1, \ldots, q-1$. So proceeding this way in decreasing fashion from $\tau_{i^{*}}$ to $\tau_{1}$, and then in increasing fashion from $\tau_{i^{*}}$ to $\tau_{q}$, ensures that the re-estimated conditional quantile functions do not cross one another. As Gelfand, Smith, and Lee (1992) explain, sampling this way may not be particularly efficient, but this is more than compensated for by the ease of implementation of the Gibbs sampler.

As far as we know, this is the only way to carry out full Bayesian calculations while avoiding well-nigh impossible numerical integrations over high-dimensional sets defined by complex restrictions. Note also that our method to enforce non-crossing quantiles can be applied in any quantile regression model with endogenous or exogenous covariates, and whether Markov-switching effects are allowed for or not. In our empirical application, for instance, we estimate non-crossing quantiles with the linear QAR model as well as the MSQAR model.

\section{Monte Carlo experiments}

In this section, we examine the performance of the Gibbs sampler by means of Monte Carlo experiments. We use as data-generating process (DGP) the Garcia and Perron (1996) Markov-switching AR(2) model, given as

$$
y_{t}=\mu\left(s_{t}\right)+\phi_{1}\left(y_{t-1}-\mu\left(s_{t-1}\right)\right)+\phi_{2}\left(y_{t-2}-\mu\left(s_{t-2}\right)\right)+\sigma\left(s_{t}\right) \epsilon_{t} \text {, }
$$

where $\mu\left(s_{t}\right)=\sum_{i=1}^{3} \mu_{i} \llbracket\left[s_{t}=i\right], \sigma\left(s_{t}\right)=\sum_{i=1}^{3} \sigma_{i} \llbracket\left[s_{t}=i\right]$, and $s_{t}$ takes values in $\{1,2,3\}$ according to the outcome of a first-order Markov chain. This three-state Markov-switching model was used by Garcia and Perron (1996) to investigate the presence of regime changes in the conditional mean and variance of the quarterly U.S. real interest rate from 1960Q1 to 1990Q4 (124 observations).

We set the true values of the parameters appearing in (14) as $\boldsymbol{\mu}=(-1.5,1.3,4), \boldsymbol{\phi}=(0.05,0.05), \sigma^{2}=$ $(5.5,1.5,6.5)$, and the transition probabilities of the Markov chain are set as $p_{i j}=0.95$ when $i=j$, and $p_{i j}=0.025$ when $i \neq j$. These values correspond closely to the estimates reported by Garcia and Perron (1996) and Kim and Nelson (1999), §9.3. We examine sample sizes $T=120,240$ and three different distributions for $\epsilon_{t}$ : (i) a standard normal distribution; (ii) a Student- $t$ distribution with three degrees of freedom, standardized to have unit variance; and (iii) a gamma distribution with shape 4 and scale 1, standardized to have mean zero and unit variance.

We consider the MSQAR $(K, p)$ model in (9), correctly specified with $K=3, p=2$, and nine different quantile levels $\tau=0.1, \ldots, 0.9$. We also include a misspecified QAR(2) to assess the effect of ignoring the Markov-switching structure. The prior for $\boldsymbol{\mu}(\tau)$ is a trivariate normal with mean $\boldsymbol{\mu}+Q_{z_{t}}(\tau)$ and covariance matrix equal to 0.12 times a diagonal matrix, where $Q_{z_{t}}(\tau)$ is the standard normal quantile function. A bivariate normal distribution with mean zero and covariance matrix equal to 0.08 times a diagonal matrix is used as the prior for $\phi(\tau)$, at all $\tau$. Finally the prior for the scale parameter $\delta$ appearing in the asymmetric Laplace distribution is an $I G(0.1 / 2$, $0.1 / 2$ ) distribution, and the transition probabilities are sampled according to Appendix B.2 using a Dirichlet distribution with hyperparameters set to 0.1 . After running the Gibbs (single- and multi-move) samplers for 5000 burn-in iterations, we use the next 20,000 draws with a thinning of 2 for inference, and the simulation results are based on 400 replications of each DGP configuration. 
From the Gibbs output we obtain $\hat{s}_{1}, \ldots, \hat{s}_{T}$, the states identified a posteriori. As explained in Section 4.2 these are found from the posterior state probabilities $\operatorname{Pr}\left(s_{t}=i \mid \mathbf{y}\right)$, which are computed by averaging the Gibbs draws of the state variables. The Gibbs sampler can then be evaluated by computing the proportion of correctly classified (PCC) states as

$$
\mathrm{PCC}=\frac{1}{T} \sum_{t=1}^{T}\left[\left[\hat{s}_{t}=s_{t}\right]\right.
$$

where $s_{t}$ is the true state at time $t$.

Table 1 reports the median along with the corresponding lower 5\% and upper 95\% quantiles of the PCC statistics across the 400 replications of each DGP configuration. We see that 84 to $94 \%$ of states are correctly identified when the quantile level $\tau$ is between 0.4 and 0.6 , and the PCCs deteriorate as the quantile level becomes more extreme towards either tail. Comparing the distributions, we observe that the best performance is achieved under Student- $t$ errors. For instance, when $T=120$ and $\tau$ is in the 0.4-0.6 range, the median PCCs under normal and gamma errors are around $80 \%$, while those under the heavier-tailed Student- $t$ errors exceed $90 \%$. It is also interesting to note that increasing the sample size does not affect much the median PCC, but rather has a greater effect of the range of the estimated PCCs. As $T$ doubles from 120 to 240 under each distribution, we can see in general a narrowing of the range between the lower and upper PCC quantiles, while the median remains almost unchanged. This is the same effect also seen when comparing the single- and multi-move samplers. Indeed, the relative computational efficiency of the multi-move relative to the single-move samplers appears most importantly as a reduction in the variance of correctly identified states.

Table 1: In-sample proportion of correctly identified states under various sample sizes and error distributions: correctly specified MSQAR $(3,2)$ model.

\begin{tabular}{|c|c|c|c|c|c|c|}
\hline \multirow[b]{3}{*}{$\tau$} & \multicolumn{4}{|c|}{ Bayesian inference } & \multicolumn{2}{|c|}{ MLE inference } \\
\hline & \multicolumn{2}{|c|}{ Single-move sampler } & \multicolumn{2}{|c|}{$\overline{\text { Multi-move sampler }}$} & \multirow[b]{2}{*}{$T=120$} & \multirow[b]{2}{*}{$T=240$} \\
\hline & $T=120$ & $T=240$ & $T=120$ & $T=240$ & & \\
\hline \multicolumn{7}{|c|}{ Panel A: Normal errors } \\
\hline \multirow[t]{2}{*}{0.1} & 0.754 & 0.777 & 0.788 & 0.798 & 0.449 & 0.473 \\
\hline & $(0.296,0.949)$ & $(0.387,0.924)$ & $(0.534,0.941)$ & $(0.592,0.903)$ & $(0.190,0.720)$ & $(0.196,0.716)$ \\
\hline \multirow[t]{2}{*}{0.2} & 0.771 & 0.786 & 0.797 & 0.790 & 0.475 & 0.511 \\
\hline & $(0.322,0.966)$ & $(0.327,0.941)$ & $(0.491,0.949)$ & $(0.587,0.920)$ & $(0.177,0.791)$ & $(0.197,0.722)$ \\
\hline \multirow[t]{2}{*}{0.3} & 0.780 & 0.790 & 0.805 & 0.811 & 0.572 & 0.598 \\
\hline & $(0.314,0.941)$ & $(0.454,0.916)$ & $(0.491,0.966)$ & $(0.580,0.937)$ & $(0.267,0.845)$ & $(0.284,0.773)$ \\
\hline \multirow[t]{2}{*}{0.4} & 0.805 & 0.798 & 0.839 & 0.853 & 0.555 & 0.627 \\
\hline & $(0.500,0.932)$ & $(0.584,0.908)$ & $(0.550,0.975)$ & $(0.654,0.945)$ & $(0.261,0.854)$ & $(0.249,0.810)$ \\
\hline \multirow[t]{2}{*}{0.5} & 0.812 & 0.819 & 0.873 & 0.875 & 0.579 & 0.645 \\
\hline & $(0.559,0.941)$ & $(0.630,0.916)$ & $(0.635,0.975)$ & $(0.726,0.958)$ & $(0.269,0.886)$ & $(0.251,0.835)$ \\
\hline \multirow[t]{2}{*}{0.6} & 0.805 & 0.811 & 0.856 & 0.861 & 0.552 & 0.641 \\
\hline & $(0.406,0.949)$ & $(0.504,0.924)$ & $(0.609,0.975)$ & $(0.680,0.958)$ & $(0.267,0.880)$ & $(0.242,0.844)$ \\
\hline \multirow[t]{2}{*}{0.7} & 0.788 & 0.811 & 0.831 & 0.840 & 0.564 & 0.626 \\
\hline & $(0.364,0.958)$ & $(0.353,0.933)$ & $(0.559,0.966)$ & $(0.638,0.941)$ & $(0.269,0.836)$ & $(0.251,0.787)$ \\
\hline \multirow[t]{2}{*}{0.8} & 0.767 & 0.803 & 0.822 & 0.819 & 0.462 & 0.529 \\
\hline & $(0.336,0.966)$ & $(0.356,0.950)$ & $(0.525,0.958)$ & $(0.618,0.929)$ & $(0.174,0.782)$ & $(0.279,0.772)$ \\
\hline \multirow[t]{2}{*}{0.9} & 0.763 & 0.773 & 0.822 & 0.817 & 0.455 & 0.545 \\
\hline & $(0.303,0.966)$ & $(0.339,0.937)$ & $(0.584,0.958)$ & $(0.639,0.920)$ & $(0.207,0.740)$ & $(0.191,0.730)$ \\
\hline \multirow[t]{2}{*}{ Average } & 0.782 & 0.796 & 0.825 & 0.829 & 0.518 & 0.577 \\
\hline & $(0.377,0.946)$ & $(0.437,0.923)$ & $(0.553,0.956)$ & $(0.634,0.930)$ & $(0.230,0.817)$ & $(0.242,0.779)$ \\
\hline \multicolumn{7}{|c|}{ Panel B: Student- $t$ errors } \\
\hline \multirow[t]{2}{*}{0.1} & 0.856 & 0.845 & 0.864 & 0.869 & 0.503 & 0.493 \\
\hline & $(0.610,0.958)$ & $(0.680,0.945)$ & $(0.635,0.966)$ & $(0.706,0.941)$ & $(0.179,0.783)$ & $(0.221,0.775)$ \\
\hline \multirow[t]{2}{*}{0.2} & 0.881 & 0.874 & 0.886 & 0.888 & 0.552 & 0.601 \\
\hline & $(0.635,0.966)$ & $(0.718,0.958)$ & $(0.695,0.975)$ & $(0.744,0.958)$ & $(0.228,0.884)$ & $(0.235,0.885)$ \\
\hline \multirow[t]{2}{*}{0.3} & 0.907 & 0.908 & 0.915 & 0.917 & 0.667 & 0.675 \\
\hline & $(0.669,0.983)$ & $(0.777,0.975)$ & $(0.737,0.983)$ & $(0.811,0.975)$ & $(0.402,0.936)$ & $(0.408,0.919)$ \\
\hline \multirow[t]{2}{*}{0.4} & 0.924 & 0.929 & 0.932 & 0.935 & 0.689 & 0.743 \\
\hline & $(0.576,0.992)$ & $(0.651,0.979)$ & $(0.805,0.992)$ & $(0.861,0.979)$ & $(0.386,0.937)$ & $(0.483,0.935)$ \\
\hline \multirow[t]{2}{*}{0.5} & 0.932 & 0.941 & 0.941 & 0.945 & 0.668 & 0.765 \\
\hline & $(0.613,0.992)$ & $(0.720,0.983)$ & $(0.839,0.992)$ & $(0.874,0.987)$ & $(0.378,0.954)$ & $(0.476,0.947)$ \\
\hline
\end{tabular}




\begin{tabular}{|c|c|c|c|c|c|c|}
\hline 0.6 & $\begin{array}{r}0.941 \\
\end{array}$ & $\begin{array}{r}0.941 \\
0702 \quad 0987\end{array}$ & $\begin{array}{r}0.941 \\
(0831 \quad 0992)\end{array}$ & $\begin{array}{r}0.941 \\
0.965 \quad 097)\end{array}$ & $\begin{array}{r}0.676 \\
0375 \quad 048)\end{array}$ & $\begin{array}{r}0.719 \\
(0.501 \quad 0.949)\end{array}$ \\
\hline \multirow[t]{2}{*}{0.7} & 0.924 & 0.920 & 0.924 & 0.924 & 0.666 & 0.707 \\
\hline & $(0.686,0.992)$ & $(0.765,0.979)$ & $(0.780,0.992)$ & $(0.819,0.983)$ & $(0.380,0.932)$ & $(0.403,0.942)$ \\
\hline \multirow[t]{2}{*}{0.8} & 0.898 & 0.891 & 0.898 & 0.895 & 0.567 & 0.576 \\
\hline & $(0.703,0.975)$ & $(0.760,0.971)$ & $(0.746,0.983)$ & $(0.773,0.971)$ & $(0.287,0.908)$ & $(0.314,0.915)$ \\
\hline \multirow[t]{2}{*}{0.9} & 0.873 & 0.866 & 0.881 & 0.886 & 0.499 & 0.534 \\
\hline & $(0.694,0.966)$ & $(0.706,0.954)$ & $(0.712,0.967)$ & $(0.718,0.962)$ & $(0.192,0.819)$ & $(0.244,0.870)$ \\
\hline \multirow[t]{2}{*}{ Average } & 0.904 & 0.901 & 0.909 & 0.911 & 0.609 & 0.645 \\
\hline & $(0.642,0.975)$ & $(0.719,0.964)$ & $(0.753,0.978)$ & $(0.796,0.966)$ & $(0.314,0.899)$ & $(0.366,0.908)$ \\
\hline \multicolumn{7}{|c|}{ Panel C: Gamma errors } \\
\hline \multirow[t]{2}{*}{0.1} & 0.720 & 0.739 & 0.814 & 0.813 & 0.484 & 0.535 \\
\hline & $(0.332,0.967)$ & $(0.256,0.945)$ & $(0.551,0.966)$ & $(0.563,0.929)$ & $(0.197,0.769)$ & $(0.219,0.823)$ \\
\hline \multirow[t]{2}{*}{0.2} & 0.742 & 0.775 & 0.805 & 0.811 & 0.624 & 0.632 \\
\hline & $(0.288,0.958)$ & $(0.298,0.945)$ & $(0.533,0.967)$ & $(0.546,0.937)$ & $(0.295,0.823)$ & $(0.318,0.832)$ \\
\hline \multirow[t]{2}{*}{0.3} & 0.780 & 0.794 & 0.818 & 0.828 & 0.626 & 0.652 \\
\hline & $(0.304,0.958)$ & $(0.349,0.933)$ & $(0.500,0.975)$ & $(0.538,0.950)$ & $(0.279,0.839)$ & $(0.310,0.863)$ \\
\hline \multirow[t]{2}{*}{0.4} & 0.788 & 0.803 & 0.839 & 0.845 & 0.714 & 0.723 \\
\hline & $(0.414,0.966)$ & $(0.538,0.929)$ & $(0.559,0.983)$ & $(0.613,0.962)$ & $(0.376,0.879)$ & $(0.424,0.857)$ \\
\hline \multirow[t]{2}{*}{0.5} & 0.807 & 0.814 & 0.864 & 0.876 & 0.709 & 0.748 \\
\hline & $(0.508,0.958)$ & $(0.592,0.916)$ & $(0.619,0.983)$ & $(0.701,0.962)$ & $(0.393,0.889)$ & $(0.492,0.855)$ \\
\hline \multirow[t]{2}{*}{0.6} & 0.790 & 0.805 & 0.864 & 0.878 & 0.712 & 0.727 \\
\hline & $(0.340,0.941)$ & $(0.499,0.941)$ & $(0.635,0.983)$ & $(0.706,0.962)$ & $(0.381,0.885)$ & $(0.490,0.876)$ \\
\hline \multirow[t]{2}{*}{0.7} & 0.780 & 0.786 & 0.839 & 0.849 & 0.612 & 0.710 \\
\hline & $(0.381,0.958)$ & $(0.420,0.929)$ & $(0.576,0.975)$ & $(0.660,0.946)$ & $(0.386,0.877)$ & $(0.413,0.859)$ \\
\hline \multirow[t]{2}{*}{0.8} & 0.758 & 0.771 & 0.805 & 0.815 & 0.529 & 0.617 \\
\hline & $(0.347,0.949)$ & $(0.398,0.954)$ & $(0.508,0.958)$ & $(0.609,0.929)$ & $(0.317,0.817)$ & $(0.350,0.822)$ \\
\hline \multirow[t]{2}{*}{0.9} & 0.729 & 0.754 & 0.805 & 0.807 & 0.486 & 0.493 \\
\hline & $(0.311,0.975)$ & $(0.356,0.950)$ & $(0.550,0.949)$ & $(0.609,0.916)$ & $(0.201,0.729)$ & $(0.268,0.738)$ \\
\hline \multirow[t]{2}{*}{ Average } & 0.766 & 0.782 & 0.828 & 0.835 & 0.611 & 0.641 \\
\hline & $(0.358,0.952)$ & $(0.411,0.933)$ & $(0.559,0.965)$ & $(0.616,0.938)$ & $(0.315,0.832)$ & $(0.359,0.832)$ \\
\hline
\end{tabular}

This table shows the median of the in-sample PCCs, defined in (15), across 400 replications of each DGP, while the numbers in parenthesis are the corresponding $5 \%$ and $95 \%$ quantiles.

The accuracy of estimation is further assessed by examining the deviations between the true conditional quantile $Q_{y_{t}}\left(\tau \mid y_{t-1}, y_{t-2}, s_{t}, s_{t-1}, s_{t-2} ; \theta\right)$ implied by the DGP in (14) and $Q_{y_{t}}\left(\tau \mid y_{t-1}, y_{t-2}, \hat{s}_{t}, \hat{s}_{t-1}, \hat{s}_{t-2} ; \hat{\theta}(\tau)\right)$, the estimated conditional quantile under the $\operatorname{MSQAR}(3,2)$ model. More precisely, we compute the mean absolute deviation error (MADE) ${ }^{4}$ across observations as

$$
\operatorname{MADE}=\frac{1}{T} \sum_{t=1}^{T}\left|Q_{y_{t}}\left(\tau \mid y_{t-1}, y_{t-2}, s_{t}, s_{t-1}, s_{t-2} ; \boldsymbol{\theta}\right)-Q_{y_{t}}\left(\tau \mid y_{t-1}, y_{t-2}, \hat{s}_{t}, \hat{s}_{t-1}, \hat{s}_{t-2} ; \hat{\boldsymbol{\theta}}(\tau)\right)\right|
$$

where $\hat{\boldsymbol{\theta}}(\tau)$ are the posterior means of the parameters, and the MADE statistic is computed for each DGP replication, as we did before with the PCC statistic. The results are shown in Table 2, where for each DGP configuration the entries are the median of the 400 MADEs reported with the corresponding lower $5 \%$ and upper $95 \%$ quantiles.

Table 2: In-sample MADE under various sample sizes and error distributions: correctly specified MSQAR $(3,2)$ model.

\begin{tabular}{|c|c|c|c|c|c|c|}
\hline \multirow[b]{3}{*}{$\tau$} & & & \multicolumn{2}{|c|}{ Bayesian inference } & \multicolumn{2}{|c|}{ MLE inference } \\
\hline & \multicolumn{2}{|c|}{ Single-move sampler } & \multicolumn{2}{|c|}{ Multi-move sampler } & \multirow[b]{2}{*}{$T=120$} & \multirow[b]{2}{*}{$T=240$} \\
\hline & $T=120$ & $T=240$ & $T=120$ & $T=240$ & & \\
\hline \multicolumn{7}{|c|}{ Panel A: Normal errors } \\
\hline \multirow[t]{2}{*}{0.1} & 1.045 & 0.980 & 1.008 & 0.945 & 1.411 & 1.373 \\
\hline & $(0.753,1.547)$ & $(0.702,1.341)$ & $(0.731,1.530)$ & $(0.680,1.275)$ & $(1.048,1.793)$ & $(1.106,1.661)$ \\
\hline \multirow{2}{*}{0.2} & 0.931 & 0.859 & 0.919 & 0.847 & 1.227 & 1.190 \\
\hline & $(0.637,1.524)$ & $(0.622,1.256)$ & $(0.632,1.332)$ & $(0.609,1.193)$ & $(0.925,1.558)$ & $(0.949,1.415)$ \\
\hline \multirow[t]{2}{*}{0.3} & 0.890 & 0.809 & 0.829 & 0.763 & 1.147 & 1.116 \\
\hline & $(0.516,1.434)$ & $(0.504,1.313)$ & $(0.495,1.317)$ & $(0.494,1.077)$ & $(0.894,1.451)$ & $(0.894,1.314)$ \\
\hline \multirow[t]{2}{*}{0.4} & 0.828 & 0.758 & 0.647 & 0.622 & 1.123 & 1.089 \\
\hline & $(0.353,1.425)$ & $(0.418,1.353)$ & $(0.346,1.162)$ & $(0.376,0.984)$ & $(0.877,1.391)$ & $(0.883,1.287)$ \\
\hline
\end{tabular}




\begin{tabular}{|c|c|c|c|c|c|c|}
\hline \multirow[t]{2}{*}{0.5} & 0.861 & 0.774 & 0.544 & 0.514 & 1.127 & 1.085 \\
\hline & $(0.298,1.473)$ & $(0.355,1.421)$ & $(0.235,1.083)$ & $(0.287,0.856)$ & $(0.873,1.409)$ & $(0.882,1.279)$ \\
\hline \multirow[t]{2}{*}{0.6} & 0.867 & 0.735 & 0.650 & 0.609 & 1.150 & 1.103 \\
\hline & $(0.385,1.466)$ & $(0.388,1.374)$ & $(0.360,1.164)$ & $(0.352,0.949)$ & $(0.885,1.431)$ & $(0.865,1.324)$ \\
\hline \multirow[t]{2}{*}{0.7} & 0.910 & 0.828 & 0.827 & 0.769 & 1.205 & 1.152 \\
\hline & $(0.538,1.511)$ & $(0.546,1.286)$ & $(0.519,1.280)$ & $(0.522,1.051)$ & $(0.909,1.580)$ & $(0.885,1.409)$ \\
\hline \multirow[t]{2}{*}{0.8} & 0.993 & 0.915 & 0.975 & 0.902 & 1.306 & 1.243 \\
\hline & $(0.699,1.557)$ & $(0.661,1.206)$ & $(0.668,1.469)$ & $(0.639,1.137)$ & $(0.971,1.630)$ & $(0.945,1.522)$ \\
\hline \multirow[t]{2}{*}{0.9} & 1.113 & 1.046 & 1.092 & 1.028 & 1.555 & 1.470 \\
\hline & $(0.829,1.633)$ & $(0.782,1.306)$ & $(0.796,1.545)$ & $(0.775,1.274)$ & $(1.130,2.040)$ & $(1.103,1.830)$ \\
\hline \multirow[t]{2}{*}{ Average } & 0.937 & 0.856 & 0.832 & 0.777 & 1.250 & 1.202 \\
\hline & $(0.556,1.503)$ & $(0.553,1.313)$ & $(0.531,1.316)$ & $(0.526,1.083)$ & $(0.975,1.618)$ & $(0.973,1.475)$ \\
\hline \multicolumn{7}{|c|}{ Panel B: Student- $t$ errors } \\
\hline \multirow[t]{2}{*}{0.1} & 0.816 & 0.777 & 0.801 & 0.752 & 1.255 & 1.186 \\
\hline & $(0.559,1.289)$ & $(0.511,1.088)$ & $(0.533,1.282)$ & $(0.499,1.040)$ & $(0.755,1.975)$ & $(0.708,1.877)$ \\
\hline \multirow[t]{2}{*}{0.2} & 0.615 & 0.592 & 0.604 & 0.569 & 1.060 & 0.987 \\
\hline & $(0.372,1.049)$ & $(0.358,0.849)$ & $(0.343,1.022)$ & $(0.349,0.832)$ & $(0.596,1.836)$ & $(0.589,1.821)$ \\
\hline \multirow[t]{2}{*}{0.3} & 0.470 & 0.439 & 0.444 & 0.427 & 0.969 & 0.909 \\
\hline & $(0.245,0.899)$ & $(0.237,0.694)$ & $(0.240,0.795)$ & $(0.230,0.643)$ & $(0.504,1.617)$ & $(0.478,1.523)$ \\
\hline \multirow[t]{2}{*}{0.4} & 0.381 & 0.336 & 0.336 & 0.316 & 0.949 & 0.958 \\
\hline & $(0.178,0.901)$ & $(0.174,0.873)$ & $(0.173,0.651)$ & $(0.173,0.519)$ & $(0.450,1.691)$ & $(0.430,1.683)$ \\
\hline \multirow[t]{2}{*}{0.5} & 0.324 & 0.302 & 0.290 & 0.278 & 1.017 & 0.990 \\
\hline & $(0.143,0.841)$ & $(0.160,0.923)$ & $(0.143,0.589)$ & $(0.146,0.467)$ & $(0.777,1.292)$ & $(0.794,1.210)$ \\
\hline \multirow[t]{2}{*}{0.6} & 0.359 & 0.322 & 0.340 & 0.298 & 1.036 & 1.011 \\
\hline & $(0.160,0.847)$ & $(0.150,0.861)$ & $(0.154,0.627)$ & $(0.146,0.525)$ & $(0.773,1.307)$ & $(0.794,1.246)$ \\
\hline \multirow[t]{2}{*}{0.7} & $0.457(0.288$ & 0.420 & 0.430 & 0.397 & 1.089 & 1.063 \\
\hline & $0.900)$ & $(0.219,0.746)$ & $(0.214,0.754)$ & $(0.210,0.671)$ & $(0.795,1.379)$ & $(0.815,1.307)$ \\
\hline \multirow[t]{2}{*}{0.8} & 0.609 & 0.569 & 0.594 & 0.549 & 1.200 & 1.170 \\
\hline & $(0.372,0.988)$ & $(0.333,0.875)$ & $(0.356,0.940)$ & $(0.331,0.838)$ & $(0.853,1.592)$ & $(0.534,2.427)$ \\
\hline \multirow[t]{2}{*}{0.9} & 0.853 & 0.801 & 0.824 & 0.773 & 1.288 & 1.090 \\
\hline & $(0.570,1.388)$ & $(0.540,1.104)$ & $(0.531,1.275)$ & $(0.526,1.061)$ & $(0.732,2.452)$ & $(0.695,1.872)$ \\
\hline \multirow[t]{2}{*}{ Average } & 0.542 & 0.506 & 0.518 & 0.484 & 1.096 & 1.041 \\
\hline & $(0.320,1.005)$ & $(0.298,0.885)$ & $(0.298,0.877)$ & $(0.29,0.728)$ & $(0.726,1.716)$ & $(0.679,1.698)$ \\
\hline \multicolumn{7}{|c|}{ Panel C: Gamma errors } \\
\hline \multirow[t]{2}{*}{0.1} & 0.769 & 0.682 & 0.769 & 0.670 & 1.247 & 1.152 \\
\hline & $(0.542,1.075)$ & $(0.467,0.931)$ & $(0.527,1.048)$ & $(0.466,0.917)$ & $(0.574,2.168)$ & $(0.493,2.110)$ \\
\hline 0.2 & 0.802 & 0.721 & 0.797 & 0.711 & 1.101 & 1.039 \\
\hline & $(0.572,1.144)$ & $(0.507,1.037)$ & $(0.544,1.076)$ & $(0.500,0.953)$ & $(0.577,1.951)$ & $(0.512,1.791)$ \\
\hline 0.3 & 0.824 & 0.754 & 0.785 & 0.714 & 1.080 & 1.059 \\
\hline & $(0.560,1.396)$ & $(0.493,1.153)$ & $(0.512,1.124)$ & $(0.475,0.958)$ & $(0.630,1.737)$ & $(0.804,1.273)$ \\
\hline 0.4 & 0.853 & 0.799 & 0.745 & 0.666 & 1.080 & 1.039 \\
\hline & $(0.512,1.437)$ & $(0.475,1.353)$ & $(0.449,1.082)$ & $(0.413,0.960)$ & $(0.822,1.350)$ & $(0.810,1.252)$ \\
\hline 0.5 & 0.888 & 0.840 & 0.632 & 0.561 & 1.087 & 1.036 \\
\hline & $(0.381,1.467)$ & $(0.364,1.481)$ & $(0.347,1.006)$ & $(0.312,0.879)$ & $(0.825,1.354)$ & $(0.804,1.255)$ \\
\hline 0.6 & 0.877 & 0.822 & 0.575 & 0.508 & 1.109 & 1.057 \\
\hline & $(0.322,1.452)$ & $(0.322,1.377)$ & $(0.237,1.033)$ & $(0.266,0.879)$ & $(0.857,1.394)$ & $(0.819,1.292)$ \\
\hline 0.7 & 0.889 & 0.810 & 0.716 & 0.677 & 1.158 & 1.106 \\
\hline & $(0.450,1.419)$ & $(0.429,1.336)$ & $(0.386,1.237)$ & $(0.408,1.022)$ & $(0.871,1.480)$ & $(0.834,1.363)$ \\
\hline 0.8 & 1.080 & 0.976 & 1.021 & 0.923 & 1.281 & 1.211 \\
\hline & $(0.700,1.603)$ & $(0.624,1.394)$ & $(0.628,1.534)$ & $(0.596,1.272)$ & $(0.939,1.639)$ & $(0.888,1.493)$ \\
\hline 0.9 & 1.349 & 1.244 & 1.304 & 1.203 & 1.582 & 1.464 \\
\hline & $(0.937,1.874)$ & $(0.852,1.687)$ & $(0.911,1.870)$ & $(0.823,1.572)$ & $(1.144,2.119)$ & $(1.051,1.813)$ \\
\hline Average & 0.925 & 0.849 & 0.816 & 0.737 & 1.192 & 1.129 \\
\hline & $(0.552,1.424)$ & $(0.503,1.301)$ & $(0.504,1.218)$ & $(0.473,1.041)$ & $(0.840,1.720)$ & $(0.812,1.551)$ \\
\hline
\end{tabular}

This table reports the median of the in-sample MADEs, defined in (16), across DGP replications. The numbers in parenthesis are the lower $5 \%$ and upper $95 \%$ quantiles of the 400 corresponding MADEs.

We see that using the asymmetric Laplace distribution as the likelihood achieves the greatest estimation precision as defined by (16) for the central quantiles under Student- $t$ errors. In this case, the MADEs are around 0.30, while they exceed 0.50 under normal and gamma distributed errors, and sometimes by far. As expected, the MADEs appear roughly symmetric under normal and Student- $t$ errors, i.e. the estimated MADEs are about the same whether $\tau$ equals 0.1 or $0.9,0.2$ or 0.8 , etc. On the contrary, the MADEs for the gamma distribution are consistently higher in the right tail, e.g. when $\tau=0.9$. This happens because the distribution is skewed to the right, meaning that there are relatively fewer observations in the right tail compared to the left tail. 
Of course, increasing the sample size improves the estimation precision at all quantile levels $\tau$. This is readily seen from the upper $95 \%$ MADE quantiles which tend to decrease as T doubles from 120 to 240 . Observe further that the multi-move sampler appears preferable, since these upper $95 \%$ limits are systematically lower than with the single-move sampler. We therefore leave aside the single-move sampler and proceed to the empirical application with the computationally more efficient multi-move sampler.

Table 3 provides a comparison of the in-sample MADEs of the misspecified QAR(2) model, obtained by Gibbs sampling and MLE. ${ }^{5}$ The pattern is clear: even with the misspecified QAR(2) model, the Bayesian approach yields smaller in-sample median MADEs relative to the MLE approach. Focusing on the averages, we see that the pattern holds for each $T$ and error distribution.

Table 3: In-sample MADE under various sample sizes and error distributions: misspecified QAR(2) model.

\begin{tabular}{|c|c|c|c|c|}
\hline \multirow[b]{2}{*}{$\tau$} & \multicolumn{2}{|r|}{ Bayesian inference } & \multicolumn{2}{|r|}{ MLE inference } \\
\hline & $T=120$ & $T=240$ & $T=120$ & $T=240$ \\
\hline 0.1 & $1.431(0.914,2.227)$ & $1.359(0.832,2.117)$ & $1.592(1.167,2.487)$ & $1.581(1.224,2.371)$ \\
\hline 0.2 & $1.256(0.793,2.017)$ & $1.269(0.813,2.016)$ & $1.392(1.060,2.193)$ & $1.376(1.068,2.097)$ \\
\hline 0.3 & $1.213(0.705,1.973)$ & $1.218(0.766,1.935)$ & $1.320(1.055,2.089)$ & $1.307(1.047,2.025)$ \\
\hline 0.4 & $1.237(0.718,2.001)$ & $1.235(0.728,1.999)$ & $1.296(1.036,2.060)$ & $1.281(1.030,2.002)$ \\
\hline 0.5 & $1.262(0.659,2.184)$ & $1.259(0.733,2.083)$ & $1.297(1.020,2.263)$ & $1.279(1.038,1.990)$ \\
\hline 0.6 & $1.324(0.709,2.308)$ & $1.340(0.713,2.630)$ & $1.324(1.020,2.409)$ & $1.298(1.048,2.445)$ \\
\hline 0.7 & $1.352(0.759,2.551)$ & $1.384(0.773,2.548)$ & $1.379(1.049,2.671)$ & $1.346(1.062,2.410)$ \\
\hline 0.8 & $1.366(0.826,2.540)$ & $1.360(0.770,2.553)$ & $1.483(1.110,2.711)$ & $1.449(1.077,2.646)$ \\
\hline 0.9 & $1.654(0.897,3.270)$ & $1.521(0.829,3.072)$ & $1.752(1.286,3.726)$ & $1.703(1.231,3.574)$ \\
\hline Average & $1.343(0.775,2.341)$ & $1.327(0.773,2.328)$ & $1.426(1.089,2.512)$ & $1.402(1.092,2.395)$ \\
\hline \multicolumn{5}{|c|}{ Panel B: Student- $t$ errors } \\
\hline 0.1 & $1.417(0.979,1.978)$ & $1.376(1.027,1.856)$ & $1.607(1.157,2.589)$ & $1.600(1.215,2.528)$ \\
\hline 0.2 & $1.173(0.856,1.510)$ & $1.147(0.902,1.457)$ & $1.348(0.994,2.174)$ & $1.345(1.068,2.130)$ \\
\hline 0.3 & $1.074(0.807,1.370)$ & $1.047(0.839,1.275)$ & $1.241(0.922,2.033)$ & $1.228(0.992,1.973)$ \\
\hline 0.4 & $1.031(0.780,1.302)$ & $1.007(0.805,1.227)$ & $1.193(0.902,1.958)$ & $1.179(0.957,1.893)$ \\
\hline 0.5 & $1.043(0.419,2.088)$ & $1.039(0.408,2.131)$ & $1.184(0.904,1.952)$ & $1.170(0.949,1.880)$ \\
\hline 0.6 & $1.146(0.413,2.634)$ & $1.105(0.437,2.393)$ & $1.204(0.918,1.963)$ & $1.191(0.972,1.912)$ \\
\hline 0.7 & $1.186(0.479,2.699)$ & $1.114(0.442,2.602)$ & $1.259(0.961,2.064)$ & $1.251(1.009,2.002)$ \\
\hline 0.8 & $1.243(0.567,2.843)$ & $1.185(0.900,1.504)$ & $1.398(1.018,2.309)$ & $1.383(1.053,2.182)$ \\
\hline 0.9 & $1.505(1.024,2.023)$ & $1.468(1.086,1.880)$ & $1.717(1.209,2.815)$ & $1.710(1.280,2.638)$ \\
\hline Average & $1.202(0.702,2.049)$ & $1.165(0.760,1.813)$ & $1.350(0.999,2.206)$ & $1.340(1.055,2.126)$ \\
\hline \multicolumn{5}{|c|}{ Panel C: Gamma errors } \\
\hline 0.1 & $1.366(0.992,1.754)$ & $1.320(0.978,1.609)$ & $1.594(1.151,2.542)$ & $1.560(1.106,2.377)$ \\
\hline 0.2 & $1.162(0.878,1.449)$ & $1.126(0.833,1.346)$ & $1.345(1.014,2.138)$ & $1.317(0.950,2.051)$ \\
\hline 0.3 & $1.106(0.845,1.386)$ & $1.088(0.573,1.896)$ & $1.278(0.984,2.055)$ & $1.248(0.939,1.974)$ \\
\hline 0.4 & $1.128(0.658,1.806)$ & $1.093(0.602,1.701)$ & $1.259(0.980,2.023)$ & $1.227(0.960,1.949)$ \\
\hline 0.5 & $1.185(0.626,2.049)$ & $1.159(0.634,1.951)$ & $1.262(0.994,2.034)$ & $1.227(0.977,1.947)$ \\
\hline 0.6 & $1.310(0.671,2.575)$ & $1.277(0.653,2.427)$ & $1.284(1.007,2.102)$ & $1.247(0.979,1.998)$ \\
\hline 0.7 & $1.362(0.720,2.507)$ & $1.398(0.720,2.631)$ & $1.338(1.000,2.172)$ & $1.294(0.988,2.067)$ \\
\hline 0.8 & $1.449(0.867,2.540)$ & $1.381(0.785,2.578)$ & $1.454(1.048,2.364)$ & $1.407(1.022,2.199)$ \\
\hline 0.9 & $1.694(1.109,2.659)$ & $1.598(1.011,2.568)$ & $1.752(1.229,2.844)$ & $1.649(1.142,2.502)$ \\
\hline Average & $1.306(0.818,2.080)$ & $1.271(0.754,2.078)$ & $1.396(1.045,2.253)$ & $1.353(1.007,2.118)$ \\
\hline
\end{tabular}

This table reports the median of the in-sample MADEs, defined in (16), across DGP replications. The numbers in parenthesis are the lower $5 \%$ and upper $95 \%$ quantiles of the 400 corresponding MADEs.

Table 4-Table 6 provide an assessment of the out-of-sample accuracy of the model forecasts. For this purpose we use the DGP in (14) to simulate trajectories of length $T+1$, and then, using only the data up to time $T$, we forecast the quantiles of $y_{T+1}$. Table 4 reports the average of $\square\left[\hat{s}_{T+1}=s_{T+1}\right]$ across DGP replications. The multimove sampler achieves the highest out-of-sample proportion of correctly identified states across sample sizes and error distributions. For instance, under normal errors, the multi-move sampler is able to identify $74.9 \%$ of states at time $T+1=241$ (on average across quantiles), which is higher than the single-move sampler $(69.4 \%)$ and the MLE approach (71.3\%).

Table 4: Out-of-sample proportion of correctly identified states under various sample sizes and error distributions: correctly specified MSQAR $(3,2)$ model. 


\begin{tabular}{|c|c|c|c|c|c|c|}
\hline \multirow[b]{3}{*}{$\tau$} & & & \multicolumn{2}{|c|}{ Bayesian inference } & \multicolumn{2}{|c|}{ MLE inference } \\
\hline & \multicolumn{2}{|c|}{ Single-move sampler } & \multicolumn{2}{|c|}{$\overline{\text { Multi-move sampler }}$} & \multirow[b]{2}{*}{$T=120$} & \multirow[b]{2}{*}{$T=240$} \\
\hline & $T=120$ & $T=240$ & $T=120$ & $T=240$ & & \\
\hline \multicolumn{7}{|c|}{ Panel A: Normal errors } \\
\hline 0.1 & 0.670 & 0.700 & 0.682 & 0.720 & 0.700 & 0.710 \\
\hline 0.2 & 0.650 & 0.700 & 0.698 & 0.720 & 0.708 & 0.740 \\
\hline 0.3 & 0.628 & 0.680 & 0.708 & 0.738 & 0.693 & 0.705 \\
\hline 0.4 & 0.630 & 0.660 & 0.755 & 0.762 & 0.708 & 0.740 \\
\hline 0.5 & 0.610 & 0.620 & 0.752 & 0.780 & 0.633 & 0.683 \\
\hline 0.6 & 0.622 & 0.675 & 0.742 & 0.782 & 0.668 & 0.683 \\
\hline 0.7 & 0.628 & 0.720 & 0.702 & 0.750 & 0.673 & 0.730 \\
\hline 0.8 & 0.668 & 0.730 & 0.705 & 0.742 & 0.668 & 0.703 \\
\hline 0.9 & 0.712 & 0.760 & 0.712 & 0.748 & 0.655 & 0.723 \\
\hline Average & 0.646 & 0.694 & 0.717 & 0.749 & 0.678 & 0.713 \\
\hline \multicolumn{7}{|c|}{ Panel B: Student- $t$ errors } \\
\hline 0.1 & 0.740 & 0.770 & 0.735 & 0.775 & 0.505 & 0.565 \\
\hline 0.2 & 0.745 & 0.792 & 0.770 & 0.795 & 0.563 & 0.548 \\
\hline 0.3 & 0.768 & 0.825 & 0.782 & 0.830 & 0.535 & 0.523 \\
\hline 0.4 & 0.765 & 0.825 & 0.818 & 0.862 & 0.513 & 0.550 \\
\hline 0.5 & 0.790 & 0.830 & 0.832 & 0.862 & 0.530 & 0.580 \\
\hline 0.6 & 0.812 & 0.832 & 0.840 & 0.865 & 0.560 & 0.548 \\
\hline 0.7 & 0.802 & 0.845 & 0.828 & 0.860 & 0.588 & 0.578 \\
\hline 0.8 & 0.805 & 0.825 & 0.805 & 0.828 & 0.638 & 0.585 \\
\hline 0.9 & 0.782 & 0.805 & 0.790 & 0.805 & 0.623 & 0.590 \\
\hline Average & 0.779 & 0.817 & 0.800 & 0.831 & 0.562 & 0.563 \\
\hline \multicolumn{7}{|c|}{ Panel C: Gamma errors } \\
\hline 0.1 & 0.740 & 0.740 & 0.740 & 0.770 & 0.623 & 0.625 \\
\hline 0.2 & 0.712 & 0.715 & 0.735 & 0.745 & 0.623 & 0.608 \\
\hline 0.3 & 0.658 & 0.700 & 0.748 & 0.765 & 0.578 & 0.575 \\
\hline 0.4 & 0.590 & 0.655 & 0.760 & 0.778 & 0.595 & 0.595 \\
\hline 0.5 & 0.608 & 0.630 & 0.752 & 0.768 & 0.573 & 0.600 \\
\hline 0.6 & 0.605 & 0.650 & 0.762 & 0.770 & 0.575 & 0.570 \\
\hline 0.7 & 0.622 & 0.692 & 0.728 & 0.762 & 0.585 & 0.635 \\
\hline 0.8 & 0.630 & 0.715 & 0.688 & 0.758 & 0.663 & 0.588 \\
\hline 0.9 & 0.680 & 0.750 & 0.698 & 0.755 & 0.633 & 0.660 \\
\hline Average & 0.649 & 0.694 & 0.735 & 0.763 & 0.605 & 0.606 \\
\hline
\end{tabular}

For each $T$ and $\tau$, the unconstrained MSQAR model is used to obtain an out-of-sample forecast $\hat{s}_{T+1}$ of the state at time $T+1$. This table shows the average of $\left[\left[\hat{s}_{T+1}=s_{T+1}\right]\right.$ across 400 replications of each DGP.

Table 5: Out-of-sample MADE under various sample sizes and error distributions: correctly specified MSQAR $(3,2)$ model.

\begin{tabular}{|c|c|c|c|c|c|c|}
\hline \multirow[b]{2}{*}{$\tau$} & \multicolumn{2}{|c|}{$\begin{array}{r}\text { Unconstrained Bayesian } \\
\text { inference }\end{array}$} & \multicolumn{2}{|c|}{$\begin{array}{r}\text { Constrained Bayesian } \\
\text { inference }\end{array}$} & \multicolumn{2}{|c|}{ MLE inference } \\
\hline & $T=120$ & $T=240$ & $T=120$ & $T=240$ & $T=120$ & $T=240$ \\
\hline \multicolumn{7}{|c|}{ Panel A: Normal errors } \\
\hline \multirow[t]{2}{*}{0.1} & 1.199 & 1.195 & 1.137 & 1.043 & 1.442 & 1.421 \\
\hline & $(0.389,3.422)$ & $(0.302,2.700)$ & $(0.306,2.742)$ & $(0.247,2.282)$ & $(0.609,3.065)$ & $(0.590,2.972)$ \\
\hline \multirow[t]{2}{*}{0.2} & 1.117 & 1.078 & 1.036 & 1.025 & 1.224 & 1.223 \\
\hline & $(0.421,3.016)$ & $(0.357,2.505)$ & $(0.298,2.378)$ & $(0.242,2.015)$ & $(0.475,3.021)$ & $(0.462,2.946)$ \\
\hline \multirow[t]{2}{*}{0.3} & 1.066 & 0.975 & 0.946 & 0.932 & 1.121 & 1.119 \\
\hline & $(0.462,2.063)$ & $(0.401,2.063)$ & $(0.278,1.902)$ & $(0.241,1.517)$ & $(0.403,3.012)$ & $(0.384,2.781)$ \\
\hline \multirow[t]{2}{*}{0.4} & 0.880 & 0.853 & 0.818 & 0.798 & 1.076 & 1.084 \\
\hline & $(0.283,2.186)$ & $(0.259,2.184)$ & $(0.162$ 1.924) & $(0.122,1.739)$ & $(0.329,3.093)$ & $(0.316,2.615)$ \\
\hline \multirow[t]{2}{*}{0.5} & 0.794 & 0.782 & 0.686 & 0.640 & 1.091 & 1.075 \\
\hline & $(0.236,1.682)$ & $(0.209,1.671)$ & $(0.114,1.917)$ & $(0.082,1.505)$ & $(0.296,3.042)$ & $(0.281,2.627)$ \\
\hline \multirow[t]{2}{*}{0.6} & 0.939 & 0.774 & 0.792 & 0.701 & 1.141 & 1.074 \\
\hline & $(0.327,2.142)$ & $(0.269,2.122)$ & $(0.151,2.086)$ & $(0.113,2.062)$ & $(0.354,3.061)$ & $(0.335,2.689)$ \\
\hline \multirow[t]{2}{*}{0.7} & 1.109 & 0.926 & 0.955 & 0.848 & 1.215 & 1.118 \\
\hline & $(0.555,2.264)$ & $(0.491,2.174)$ & $(0.220,2.204)$ & $(0.193,2.155)$ & $(0.462,3.071)$ & $(0.446,2.820)$ \\
\hline
\end{tabular}




\begin{tabular}{|c|c|c|c|c|c|c|}
\hline 0.8 & $\begin{array}{r}1.233 \\
(0.598,2.354)\end{array}$ & $\begin{array}{r}1.002 \\
(0.539,2.344)\end{array}$ & $\begin{array}{r}1.104 \\
(0.303,2.277)\end{array}$ & $\begin{array}{r}0.992 \\
(0.277,1.895)\end{array}$ & $\begin{array}{r}1.333 \\
(0.499,3.045)\end{array}$ & $\begin{array}{r}1.203 \\
(0.481,2.909)\end{array}$ \\
\hline \multirow[t]{2}{*}{0.9} & 1.376 & 1.154 & 1.211 & 1.091 & 1.635 & 1.439 \\
\hline & $(0.551,2.909)$ & $(0.483,2.479)$ & $(0.252,2.762)$ & $(0.218,2.387)$ & $(0.572,3.078)$ & $(0.560,2.975)$ \\
\hline \multirow[t]{2}{*}{ Average } & 1.079 & 0.971 & 0.965 & 0.897 & 1.253 & 1.195 \\
\hline & $(0.424,2.344)$ & $(0.368,2.129)$ & $(0.231,2.265)$ & $(0.193,1.950)$ & $(0.451,3.047)$ & $(0.435,2.824)$ \\
\hline \multicolumn{7}{|c|}{ Panel B: Student- $t$ errors } \\
\hline \multirow{2}{*}{0.1} & 1.134 & 0.948 & 0.948 & 0.838 & 1.401 & 1.288 \\
\hline & $(0.511,3.082)$ & $(0.423,2.752)$ & $(0.232,2.395)$ & $(0.181,2.206)$ & $(0.514,2.975)$ & $(0.499,2.902)$ \\
\hline \multirow[t]{2}{*}{0.2} & 0.885 & 0.761 & 0.752 & 0.644 & 1.207 & 1.181 \\
\hline & $(0.446,2.359)$ & $(0.354,1.906)$ & $(0.279,1.870)$ & $(0.230,1.223)$ & $(0.464,3.087)$ & $(0.446,3.027)$ \\
\hline \multirow[t]{2}{*}{0.3} & 0.824 & 0.637 & 0.631 & 0.498 & 1.094 & 1.096 \\
\hline & $(0.424,1.956)$ & $(0.368,1.926)$ & $(0.208,1.851)$ & $(0.167,1.193)$ & $(0.435,3.018)$ & $(0.415,2.973)$ \\
\hline \multirow[t]{2}{*}{0.4} & 0.718 & 0.526 & 0.509 & 0.384 & 1.067 & 1.034 \\
\hline & $(0.298,1.887)$ & $(0.230,1.815)$ & $(0.131,1.825)$ & $(0.102,1.478)$ & $(0.300,2.948)$ & $(0.286,2.833)$ \\
\hline \multirow[t]{2}{*}{0.5} & 0.669 & 0.528 & 0.454 & 0.305 & 1.093 & 1.042 \\
\hline & $(0.307,1.796)$ & $(0.254,1.416)$ & $(0.130,1.791)$ & $(0.079,1.419)$ & $(0.344,2.860)$ & $(0.327,2.830)$ \\
\hline \multirow[t]{2}{*}{0.6} & 0.639 & 0.540 & 0.461 & 0.334 & 1.122 & 1.071 \\
\hline & $(0.222,1.706)$ & $(0.152,1.606)$ & $(0.118,1.876)$ & $(0.070,1.448)$ & $(0.350,2.978)$ & $(0.332,2.873)$ \\
\hline \multirow[t]{2}{*}{0.7} & 0.721 & 0.616 & 0.552 & 0.408 & 1.166 & 1.135 \\
\hline & $(0.494,2.180)$ & $(0.459,1.688)$ & $(0.241,1.839)$ & $(0.189,1.227)$ & $(0.424,3.041)$ & $(0.414,2.988)$ \\
\hline \multirow[t]{2}{*}{0.8} & 0.868 & 0.760 & 0.699 & 0.561 & 1.289 & 1.252 \\
\hline & $(0.470,2.623)$ & $(0.432,1.949)$ & $(0.260,1.995)$ & $(0.218,1.175)$ & $(0.494,2.985)$ & $(0.479,2.927)$ \\
\hline \multirow[t]{2}{*}{0.9} & 1.116 & 0.981 & 0.991 & 0.809 & 1.386 & 1.266 \\
\hline & $(0.506,3.209)$ & $(0.436,2.753)$ & $(0.217,2.736)$ & $(0.183,2.335)$ & $(0.609,2.968)$ & $(0.597,2.912)$ \\
\hline \multirow[t]{2}{*}{ Average } & 0.842 & 0.699 & 0.666 & 0.531 & 1.203 & 1.152 \\
\hline & $(0.409,2.266)$ & $(0.345,1.979)$ & $(0.202,2.019)$ & $(0.158,1.522)$ & $(0.453,2.940)$ & $(0.438,2.877)$ \\
\hline \multicolumn{7}{|c|}{ Panel C: Gamma errors } \\
\hline \multirow[t]{2}{*}{0.1} & 0.882 & 0.802 & 0.763 & 0.688 & 1.248 & 1.235 \\
\hline & $(0.449,2.542)$ & $(0.370,1.742)$ & $(0.211,0.915)$ & $(0.176,0.466)$ & $(0.606,2.828)$ & $(0.590,2.803)$ \\
\hline \multirow[t]{2}{*}{0.2} & 0.888 & 0.836 & 0.794 & 0.731 & 1.137 & 1.069 \\
\hline & $(0.627,2.298)$ & $(0.543,1.531)$ & $(0.285,0.902)$ & $(0.231,0.516)$ & $(0.507,2.671)$ & $(0.495,2.601)$ \\
\hline \multirow[t]{2}{*}{0.3} & 0.906 & 0.811 & 0.797 & 0.738 & 1.071 & 1.043 \\
\hline & $(0.435,1.764)$ & $(0.366,1.753)$ & $(0.312,0.945)$ & $(0.266,0.410)$ & $(0.351,2.752)$ & $(0.339,2.712)$ \\
\hline \multirow[t]{2}{*}{0.4} & 0.856 & 0.795 & 0.803 & 0.768 & 1.072 & 1.027 \\
\hline & $(0.392,2.063)$ & $(0.334,1.892)$ & $(0.161,1.956)$ & $(0.110,1.779)$ & $(0.369,2.553)$ & $(0.356,2.488)$ \\
\hline \multirow[t]{2}{*}{0.5} & 0.846 & 0.810 & 0.727 & 0.710 & 1.083 & 1.034 \\
\hline & $(0.305,2.313)$ & $(0.235,2.113)$ & $(0.163,1.900)$ & $(0.113,1.490)$ & $(0.247,2.705)$ & $(0.235,2.605)$ \\
\hline \multirow[t]{2}{*}{0.6} & 0.822 & 0.776 & 0.650 & 0.667 & 1.076 & 1.078 \\
\hline & $(0.289,2.064)$ & $(0.200,1.638)$ & $(0.178,1.732)$ & $(0.123,1.287)$ & $(0.429,2.679)$ & $(0.418,2.624)$ \\
\hline \multirow[t]{2}{*}{0.7} & 1.001 & 0.891 & 0.839 & 0.800 & 1.132 & 1.136 \\
\hline & $(0.437,2.199)$ & $(0.359,1.990)$ & $(0.282,2.172)$ & $(0.229,1.827)$ & $(0.483,2.843)$ & $(0.462,2.738)$ \\
\hline 0.8 & 1.289 & 1.052 & 1.139 & 1.016 & 1.263 & 1.267 \\
\hline & $(0.397,2.562)$ & $(0.306,2.526)$ & $(0.294,2.593)$ & $(0.247,2.208)$ & $(0.512,3.114)$ & $(0.473,3.041)$ \\
\hline 0.9 & 1.501 & 1.378 & 1.402 & 1.302 & 1.540 & 1.525 \\
\hline & $(0.631,3.798)$ & $(0.578,3.228)$ & $(0.257,3.204)$ & $(0.220,2.909)$ & $(0.565,2.998)$ & $(0.503,2.963)$ \\
\hline Average & 0.999 & 0.906 & 0.879 & 0.824 & 1.180 & 1.157 \\
\hline & $(0.440,2.379)$ & $(0.366,2.045)$ & $(0.238,1.813)$ & $(0.191,1.432)$ & $(0.422,2.806)$ & $(0.398,2.739)$ \\
\hline
\end{tabular}

For each $T$, the MSQAR model is used to obtain an out-of-sample forecast of the $\tau$ th quantile of $y_{T+1}$. This table reports the median of the out-of-sample MADEs across DGP replications. The numbers in parenthesis are the lower $5 \%$ and upper $95 \%$ quantiles of the 400 corresponding MADEs. The Bayesian inferences are based on the multi-move sampler.

Table 6: Out-of-sample MADE under various sample sizes and error distributions: misspecified QAR(2) model.

\begin{tabular}{|c|c|c|c|c|}
\hline \multirow[b]{2}{*}{$\tau$} & \multicolumn{2}{|r|}{ Bayesian inference } & \multicolumn{2}{|r|}{ MLE inference } \\
\hline & $T=120$ & $T=240$ & $T=120$ & $T=240$ \\
\hline \multicolumn{5}{|c|}{ Panel A: Normal errors } \\
\hline 0.1 & $1.519(0.655,3.128)$ & $1.433(0.613,2.948)$ & $1.786(0.902,3.313)$ & $1.739(0.714,3.193)$ \\
\hline 0.2 & $1.323(0.591,2.954)$ & $1.373(0.541,2.809)$ & $1.575(0.766,3.069)$ & $1.539(0.660,2.899)$ \\
\hline 0.3 & $1.276(0.472,2.628)$ & $1.270(0.431,2.458)$ & $1.456(0.870,2.878)$ & $1.399(0.684,2.748)$ \\
\hline 0.4 & $1.280(0.423,2.686)$ & $1.353(0.374,2.476)$ & $1.395(0.850,2.811)$ & $1.365(0.688,2.741)$ \\
\hline 0.5 & $1.299(0.485,2.717)$ & $1.373(0.439,2.572)$ & $1.326(0.536,2.777)$ & $1.368(0.351,2.552)$ \\
\hline
\end{tabular}




\begin{tabular}{ccccc}
0.6 & $1.348(0.475,2.866)$ & $1.412(0.417,2.696)$ & $1.382(0.616,2.906)$ & $1.283(0.445,2.731)$ \\
0.7 & $1.447(0.610,2.650)$ & $1.439(0.556,2.410)$ & $1.494(0.793,2.875)$ & $1.398(0.684,2.765)$ \\
0.8 & $1.471(0.616,2.835)$ & $1.406(0.560,2.765)$ & $1.506(0.550,2.935)$ & $1.488(0.402,2.755)$ \\
0.9 & $1.876(0.684,3.178)$ & $1.496(0.631,2.928)$ & $1.837(0.872,3.251)$ & $1.598(0.762,3.191)$ \\
Average & $1.427(0.557,2.849)$ & $1.395(0.507,2.674)$ & $1.528(0.751,2.979)$ & $1.464(0.599,2.842)$ \\
Panel B: Student-t errors & & & \\
0.1 & $1.539(0.574,3.062)$ & $1.412(0.524,3.058)$ & $1.776(0.818,3.237)$ & $1.722(0.627,3.032)$ \\
0.2 & $1.298(0.481,2.721)$ & $1.186(0.437,2.546)$ & $1.517(0.816,2.851)$ & $1.451(0.642,2.756)$ \\
0.3 & $1.136(0.492,2.531)$ & $1.083(0.441,2.341)$ & $1.373(0.789,2.646)$ & $1.320(0.642,2.581)$ \\
0.4 & $1.102(0.493,2.427)$ & $1.052(0.436,2.287)$ & $1.311(0.596,2.627)$ & $1.267(0.492,2.472)$ \\
0.5 & $1.195(0.515,2.464)$ & $1.212(0.461,2.389)$ & $1.293(0.614,2.644)$ & $1.255(0.432,2.643)$ \\
0.6 & $1.154(0.425,2.679)$ & $1.326(0.380,2.579)$ & $1.298(0.515,2.729)$ & $1.262(0.349,2.545)$ \\
0.7 & $1.278(0.642,2.943)$ & $1.236(0.602,2.908)$ & $1.340(0.667,2.963)$ & $1.310(0.550,2.702)$ \\
0.8 & $1.422(0.625,3.099)$ & $1.348(0.569,3.054)$ & $1.479(0.820,3.209)$ & $1.442(0.680,2.744)$ \\
0.9 & $1.540(0.545,3.198)$ & $1.529(0.487,3.068)$ & $1.756(0.886,3.392)$ & $1.752(0.688,3.207)$ \\
Average & $1.296(0.528,2.796)$ & $1.265(0.497,2.674)$ & $1.460(0.716,2.911)$ & $1.420(0.565,2.732)$ \\
Panel C: Gamma error & & & \\
0.1 & $1.316(0.538,3.098)$ & $1.343(0.495,2.898)$ & $1.625(0.741,3.138)$ & $1.587(0.611,2.937)$ \\
0.2 & $1.133(0.598,2.696)$ & $1.114(0.541,2.686)$ & $1.498(0.783,2.901)$ & $1.392(0.644,2.736)$ \\
0.3 & $1.121(0.522,2.911)$ & $1.099(0.470,2.836)$ & $1.357(0.842,2.936)$ & $1.286(0.735,2.766)$ \\
0.4 & $1.158(0.378,2.841)$ & $1.165(0.329,2.651)$ & $1.277(0.487,2.996)$ & $1.227(0.329,2.948)$ \\
0.5 & $1.259(0.552,2.923)$ & $1.236(0.503,2.673)$ & $1.254(0.739,2.983)$ & $1.205(0.545,2.888)$ \\
0.6 & $1.394(0.348,2.706)$ & $1.332(0.295,2.706)$ & $1.353(0.694,2.866)$ & $1.296(0.564,2.841)$ \\
0.7 & $1.358(0.581,2.892)$ & $1.492(0.530,2.702)$ & $1.386(0.772,3.047)$ & $1.369(0.593,2.890)$ \\
0.8 & $1.401(0.608,3.050)$ & $1.373(0.556,2.993)$ & $1.493(0.649,3.130)$ & $1.432(0.544,3.044)$ \\
0.9 & $1.584(0.621,3.133)$ & $1.622(0.569,3.093)$ & $1.751(0.770,3.458)$ & $1.694(0.634,3.388)$ \\
Average & $1.303(0.516,2.922)$ & $1.308(0.464,2.808)$ & $1.443(0.708,3.059)$ & $1.387(0.564,2.966)$ \\
\hline
\end{tabular}

For each $T$, the linear QAR model is used to obtain an out-of-sample forecast of the $\tau$ th quantile of $y_{T+1}$. This table reports the median of the out-of-sample MADEs across DGP replications. The numbers in parenthesis are the lower $5 \%$ and upper $95 \%$ quantiles of the 400 corresponding MADEs.

Table 5 compares the out-of-sample MADEs obtained with the unconstrained and constrained multi-move Gibbs samplers, as well as those obtained using MLE approach. The Bayesian methods clearly dominate the MLE approach with smaller MADEs across the board. Table 5 also makes clear the gains obtained by imposing the non-crossing restriction. Indeed for each sample size and error distribution, we see the average MADEs decrease when the non-crossing restriction is imposed. When the errors are Student- $t$ for instance, the constrained sampler achieves a median MADE at time $T+1=241$ of 0.531 , which is lower than its unconstrained counterpart (0.700) and the MLE approach (1.152).

Table 6 shows the out-of-sample MADEs obtained under the misspecified QAR(2) model, estimated with the Bayesian and MLE methods. Echoing the pattern already revealed in Table 3, we see from Table 6 that the good performance of the Bayesian approach continues to hold out of sample, despite the QAR model misspecification. In light of these results, we leave aside the MLE method and move on to the empirical application with the Gibbs sampling approach. For the MSQAR, we proceed with the multi-move sampler.

\section{Empirical application}

In this section we extend the Garcia and Perron (1996) analysis of structural changes in the conditional mean and variance of the U.S. real interest rate. Garcia and Perron (1996) found that the U.S. real interest rate can be described by a Markov-switching model with three states. Specifically, their results suggest that the conditional mean and variances are different for the periods 1961-1973, 1973-1980, and 1980-1986. The proposed MSQAR model allows us to go beyond the first two moments and examine how the presence of Markov-switching regimes affects the quantiles of the conditional distribution. For instance, are the tails affected the same way as the centre of the distribution? To examine this question, we began by expanding their sample period to cover 1947Q1 to 2015Q1. A measure of the U.S. real interest rate was then constructed using quarterly data on the nominal interest rate and the consumer price index, both obtained from the FRED database at the Federal Reserve Bank of St. Louis. The resulting time series of 273 observations is plotted in Figure 1. The usefulness of the MSQAR model is examined in terms of its in-sample fit and out-of-sample forecasting ability. 


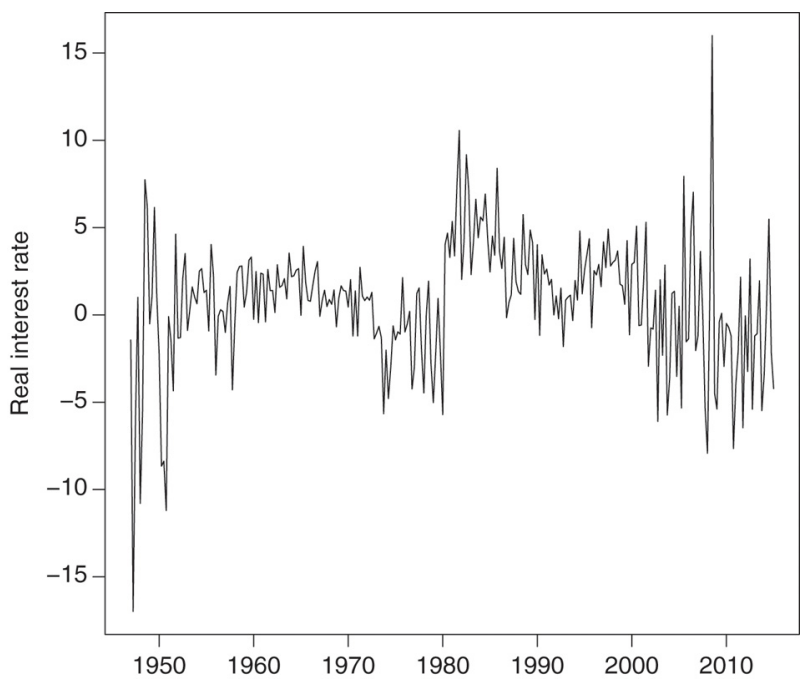

Figure 1: U.S. quarterly real interest rate from 1947Q1 to 2015Q1.

\subsection{In-sample estimation results}

We estimated the MSQAR $(K, p)$ model by letting the number of lags vary as $p=1,2,3$ and the number of regimes vary as $K=1, \ldots, 5$. Recall that when $K=1$, the MSQAR model reduces to the linear QAR $(p)$ with $\mu(\tau)=c(\tau) /\left(1-\sum_{j=1}^{p} \phi_{j}(\tau)\right)$. The models were applied at quantile levels $\tau=0.1,0.2, \ldots, 0.9$. In each case, the Gibbs sampler was used to perform the full Bayesian calculations with priors set as in the previous section. Table 7 shows the log marginal likelihood for each model at each quantile level, and the last column shows the average $\log$ marginal likelihood across the values of $\tau$. Starting with the Koenker and Xiao (2006) linear QAR specifications, we find that $p=3$ lags is generally preferable. ${ }^{6}$ Then, allowing for Markov-switching effects, we see from Table 7 that on average the marginal likelihood of the MSQAR peaks with $K=3$ and then decreases with $K=4$ and $K=5$. So holding $p=3$ lags, we find that $K=3$ regimes provides the best MSQAR specification with an average log marginal likelihood of -704 .

Table 7: Comparison of log marginal likelihoods.

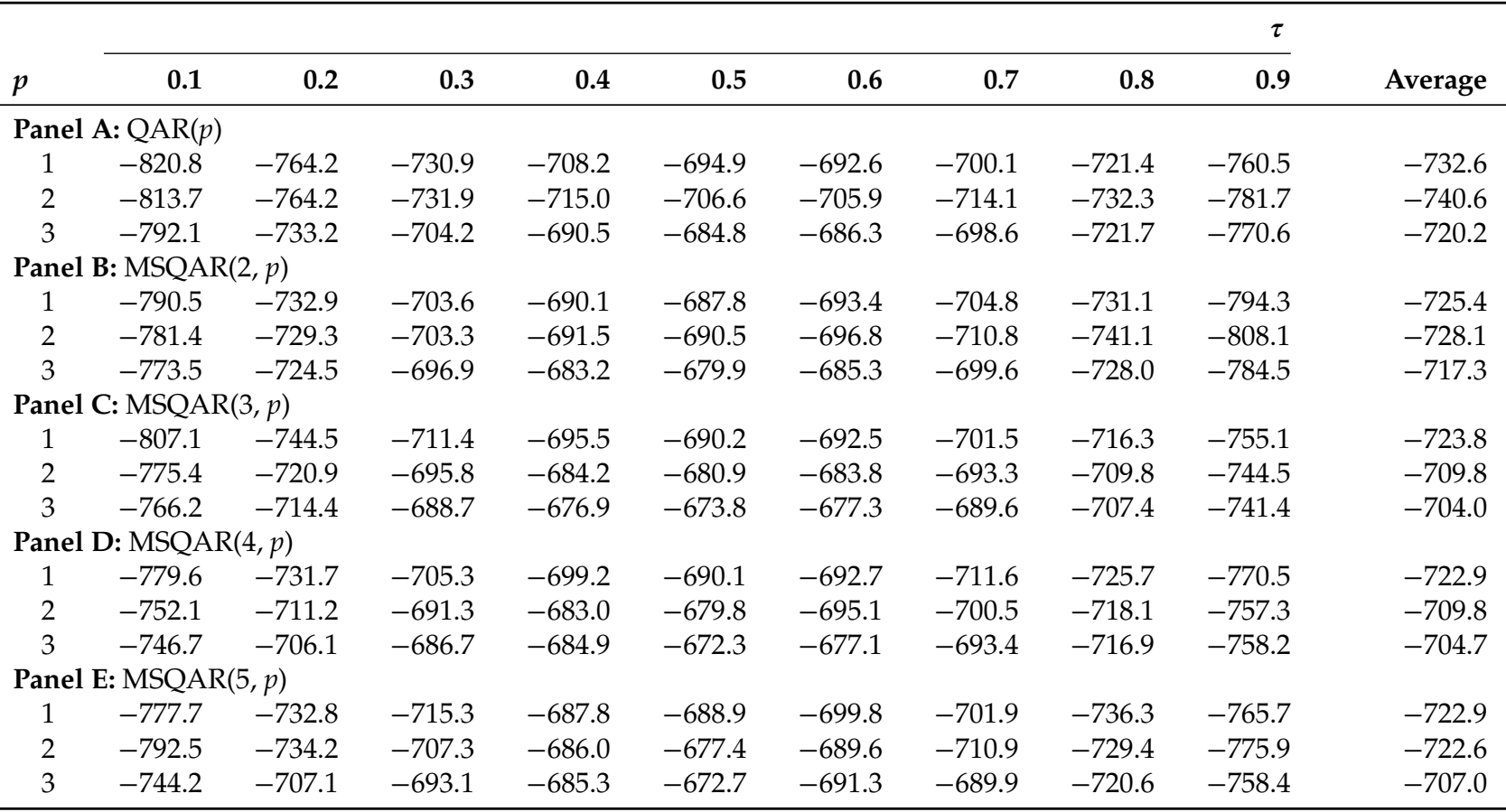

For each probability level $\tau$, this table reports the log marginal likelihood values attained by the MSQAR $(K, p)$ specification with number of regimes equal to $K=1,2,3$ and number of lags equal to $p=1,2,3$. Recall that the QAR $(p)$ corresponds to the MSQAR $(1, p)$. The last column reports the log marginal likelihood averaged across values of $\tau$. 
Table 8 presents the posterior inference results for the unconstrained MSQAR $(3,3)$ models. For each model at quantile level $\tau$, the table reports the posterior mean for each parameter, along with the corresponding numerical standard error (NSE) and the value of the Geweke (1992) test statistic. If the output of the Markov chain is compatible with stationarity, then this statistic follows a standard normal distribution. The generally insignificant values in Table 8 indicate that convergence to the stationary distribution was achieved. The estimated transition probabilities are seen to vary slightly as $\tau$ ranges from 0.1 to 0.9 . From Table 7 we see that the $\operatorname{MSQAR}(3,3)$ at $\tau=0.5$ is the best model (with a $\log$ marginal likelihood of -673.8 ) and the corresponding estimates of the state transition probabilities can be read from Table 8. So the posterior state classification (shown in Figure 2) and our stepwise re-estimation procedure proceeded with $\tau_{i^{*}}=0.5$. 


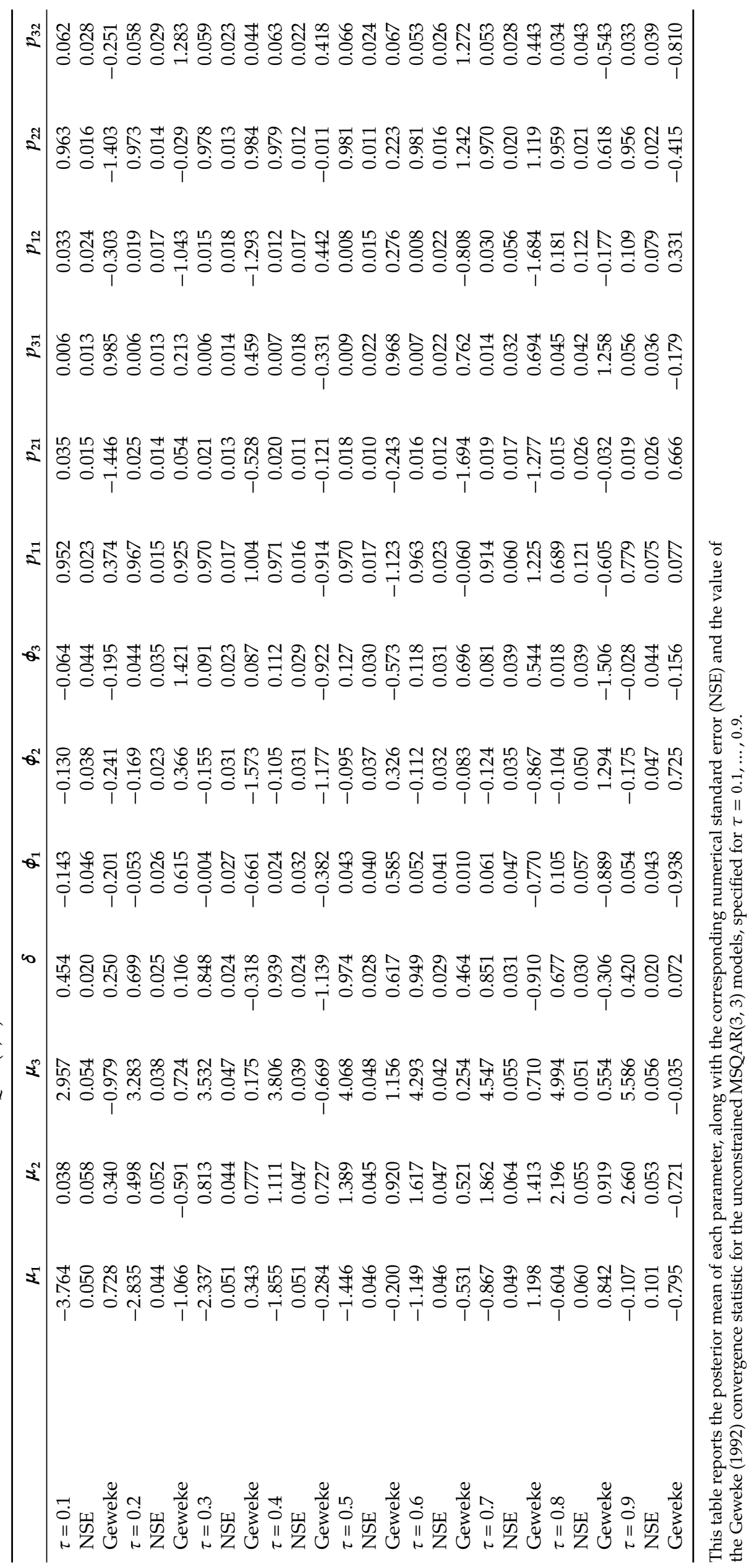




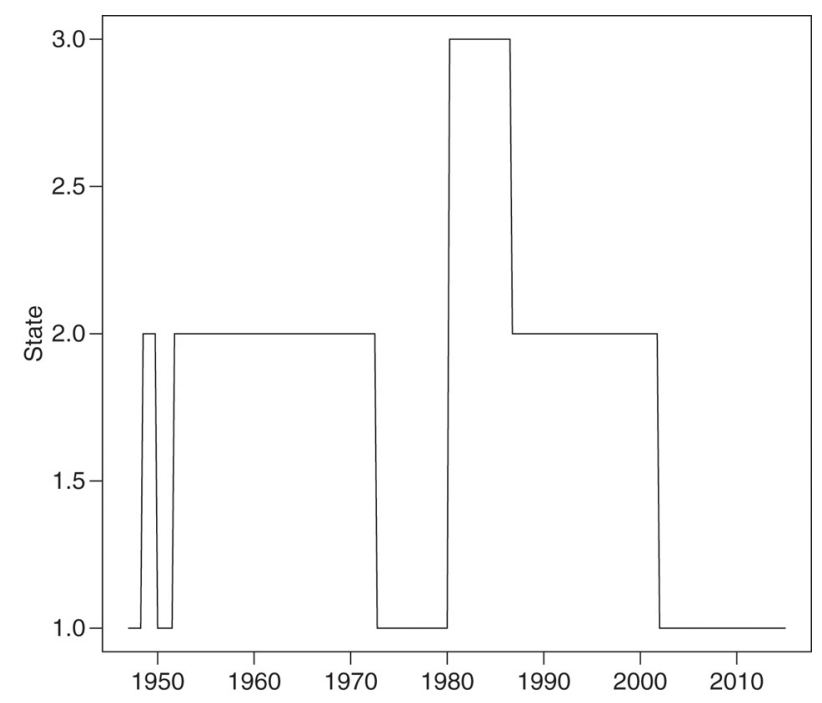

Figure 2: Posterior state classification obtained with the MSQAR $(3,3)$ model at $\tau_{i^{*}}=0.5$.

To economize on space we present a graphical comparison of the parameter estimates for the considered models. Figure 3 and Figure 4 correspond to the unconstrained and constrained versions of the QAR(3) models, respectively. We see immediately that both versions yield very similar point estimates (posterior means). The non-crossing restriction thus seems to hold fairly well under this linear specification. Note, however, that the reported $95 \%$ credible intervals appear differently when the non-crossing restriction is explicitly imposed. Indeed, the "bow-tie" pattern seen in Figure 4 (and Figure 6) simply reflects the fact that the stepwise re-estimation procedure conditions on more information as $\left|\tau_{j}-\tau_{i^{*}}\right|$ increases. The QAR(3) specification reveals a quantile regression location parameter $\mu_{1}$ that increases monotonically with $\tau$. The autoregressive parameters $\phi_{2}$ and $\phi_{3}$ suggest that the dynamics of the interest rate differ across quantile levels, even though $\phi_{1}$ itself doesn't vary much with $\tau$.
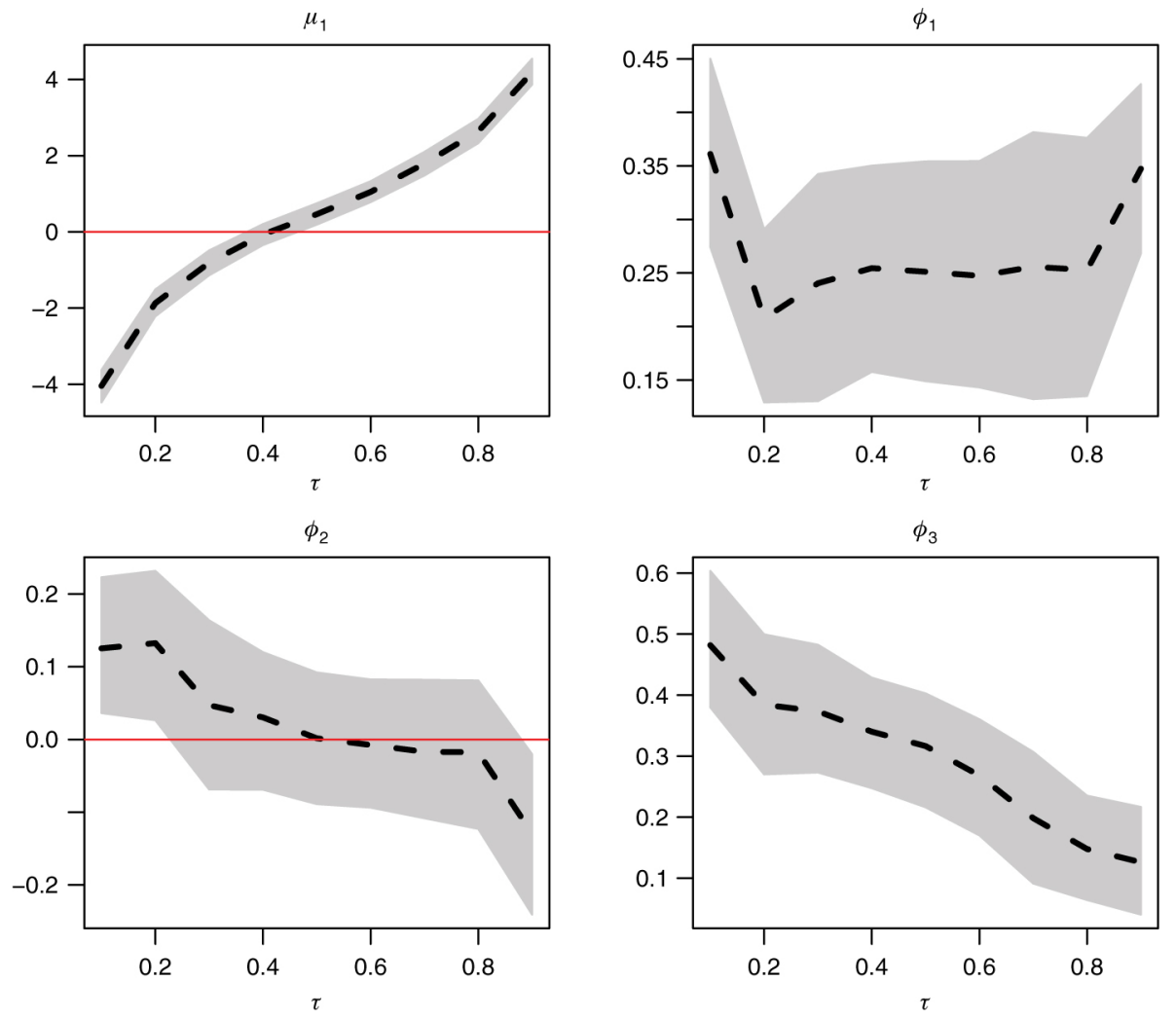

Figure 3: Unconstrained QAR(3) model parameter estimates (posterior means) across quantile probability levels $\tau$. The dashed lines connect the posterior means, while the shaded areas delimit the $95 \%$ credible intervals. 

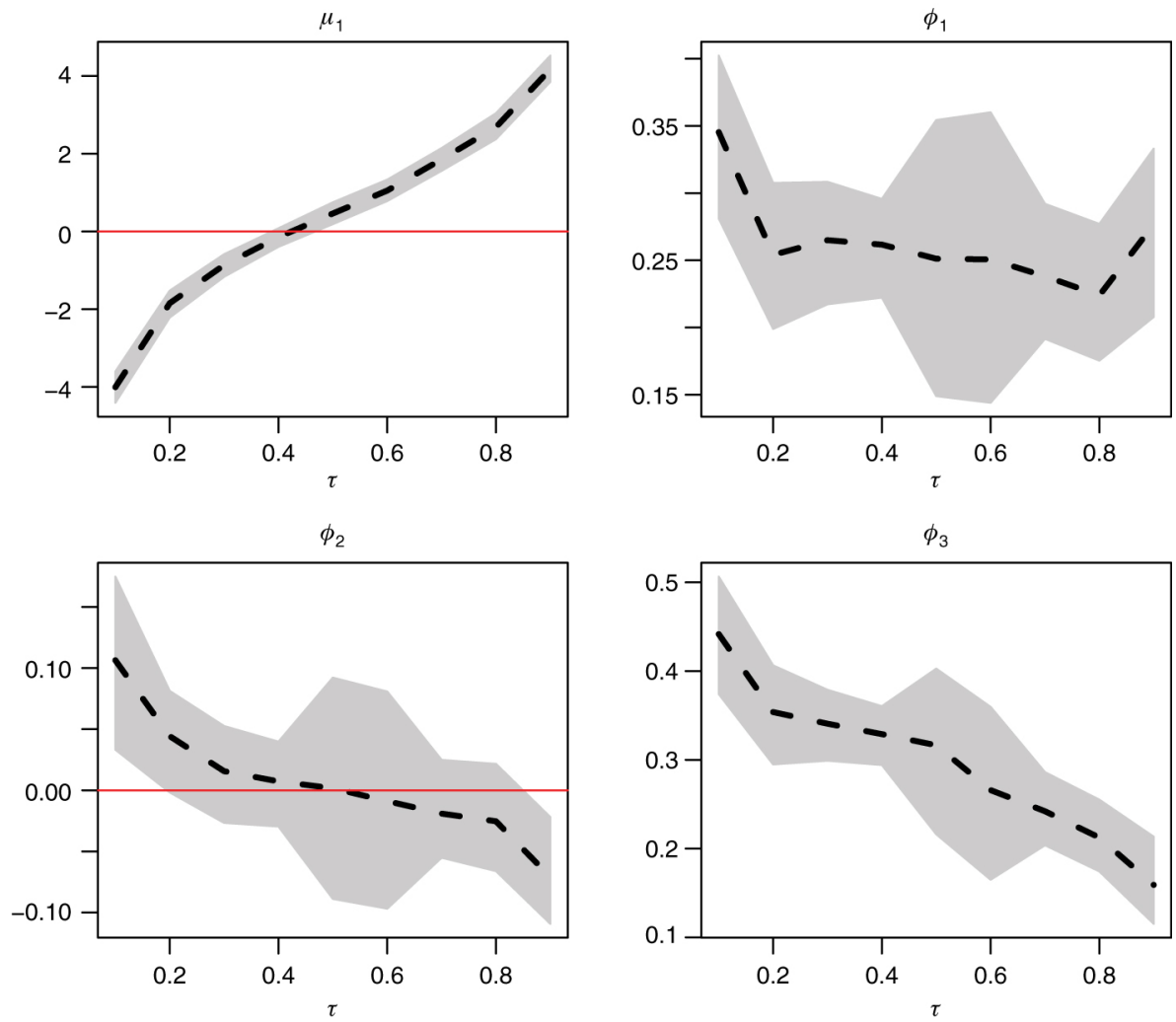

Figure 4: Non-crossing QAR(3) model parameter estimates (posterior means) across quantile probability levels $\tau$. The dashed lines connect the posterior means, while the shaded areas delimit the $95 \%$ credible intervals.

Figure 5 and Figure 6 show the estimates of $\mu_{i}$ and $\phi_{i}, i=1,2,3$, under the MSQAR $(3,3)$ specifications. In fact Figure 5 is just a graphical depiction of the information already presented in Table 8, while Figure 6 corresponds to the non-crossing version of the Markov-switching specification. We see that the estimated values of $\mu_{1}, \mu_{2}, \mu_{3}$ are well separated, which indicates that the regimes are well identified. The imposition of the noncrossing restriction clearly affects the estimates of the autoregressive parameters. The posterior mean estimates in Figure 5 reveal that: (i) $\phi_{1}$ generally increases with $\tau$; (ii) $\phi_{2}$ has no clear pattern; and (iii) $\phi_{3}$ follows an inverted U-shaped pattern. On the contrary all three estimated autoregressive parameters appear more disciplined in Figure 6, each conforming more to an inverted U-shaped pattern. 
$\mu_{1}$
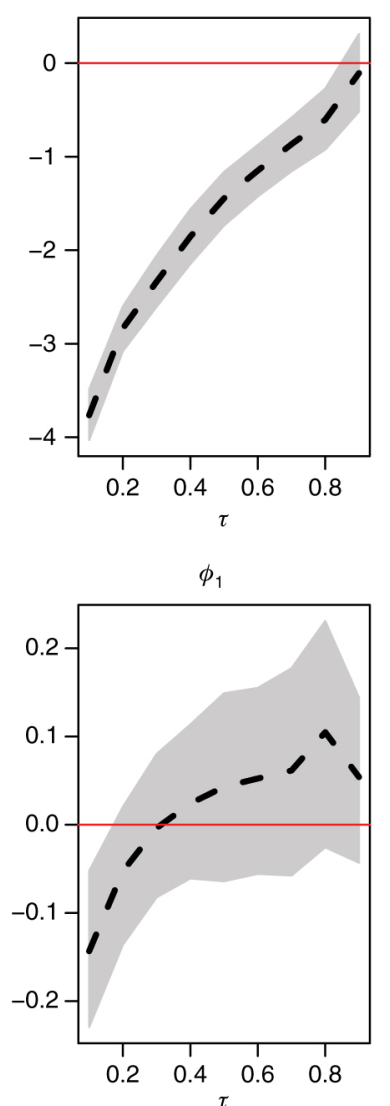

$\mu_{2}$

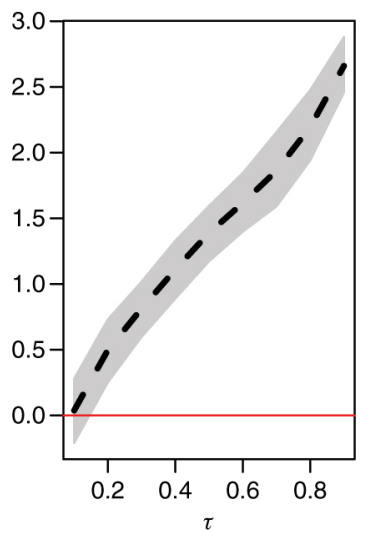

$\phi_{2}$

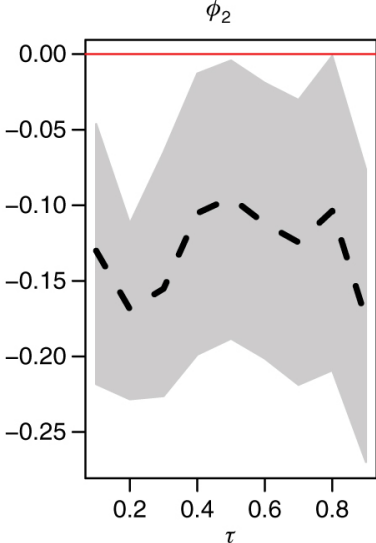

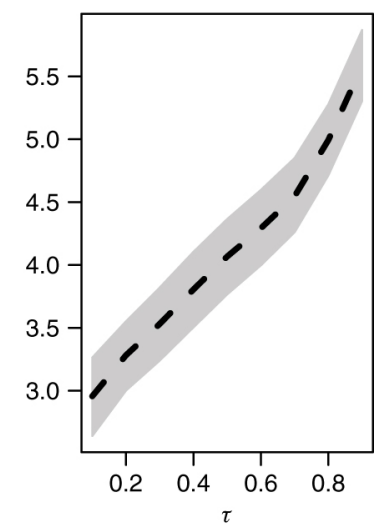

$\phi_{3}$

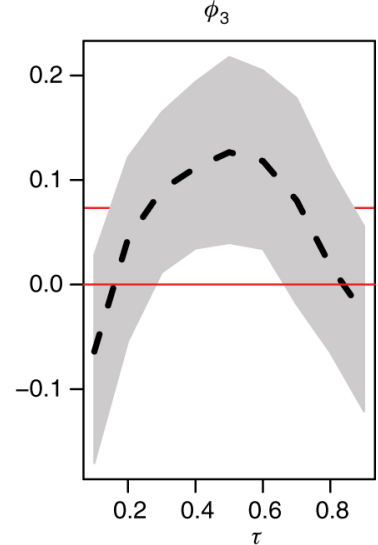

Figure 5: Unconstrained MSQAR $(3,3)$ model parameter estimates (posterior means) across quantile probability levels $\tau$. The dashed lines connect the posterior means, while the shaded areas delimit the $95 \%$ credible intervals.
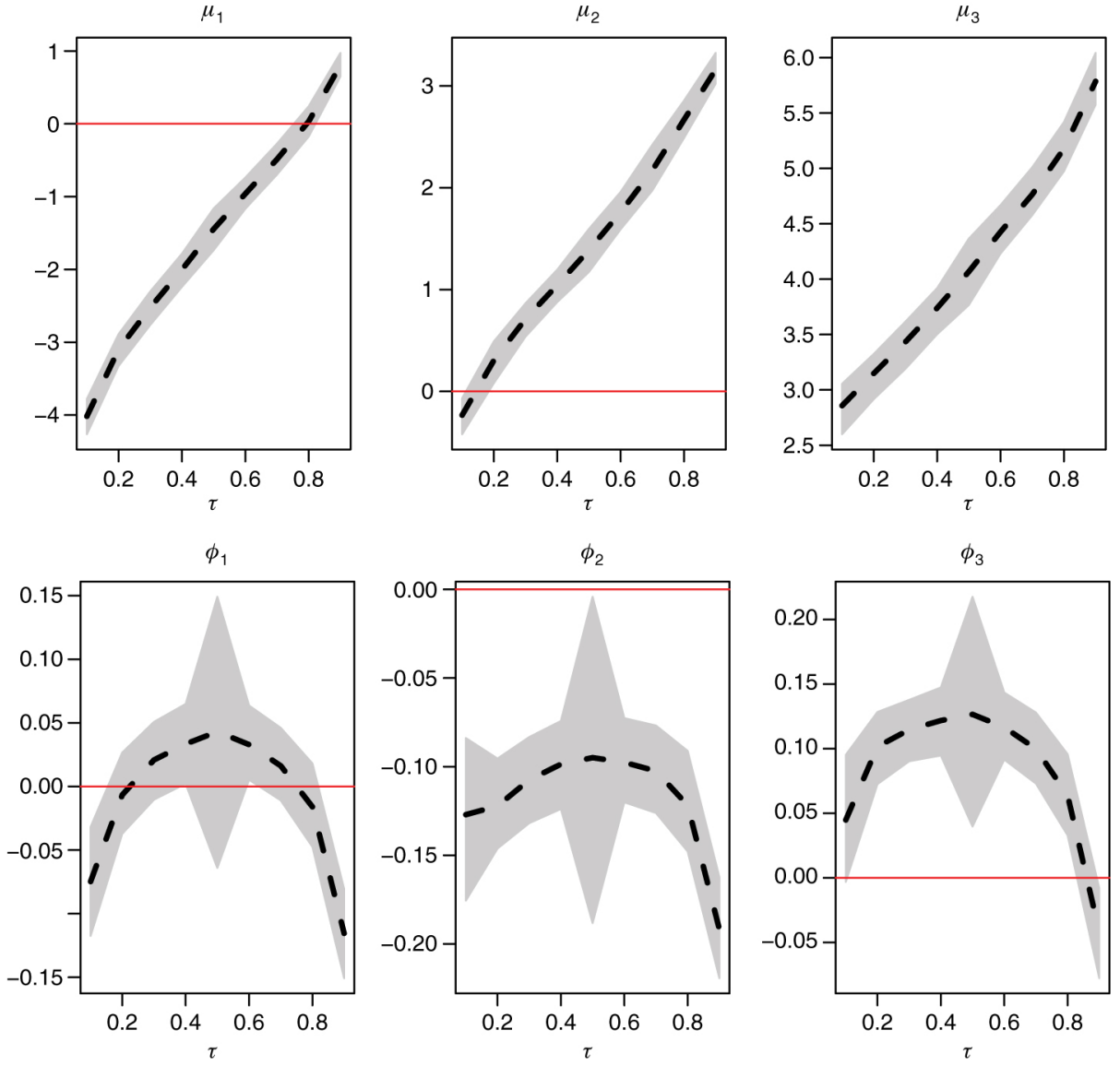
Figure 6: Non-crossing MSQAR(3, 3) model parameter estimates (posterior means) across quantile probability levels $\tau$. The dashed lines connect the posterior means, while the shaded areas delimit the $95 \%$ credible intervals.

The estimation results can also be gleaned from Figure 7-Figure 10, which show the fitted quantiles for the linear QAR and the MSQAR specifications. The unconstrained quantiles are shown in Figure 7 and Figure 9, while the constrained ones are in Figure 8 and Figure 10. Although Figure 7 and Figure 8 appear quite similar, there are in fact three occurrences of crossing quantiles in Figure 7 with the QAR models: once between the quantiles at levels 0.2 and 0.3 in 2008Q1, once between the quantiles at levels 0.5 and 0.6 in 2008Q2, and once between the quantiles at levels 0.8 and 0.9 in 2008Q1. By construction, the fitted quantiles in Figure 8 have no crossings whatsoever.

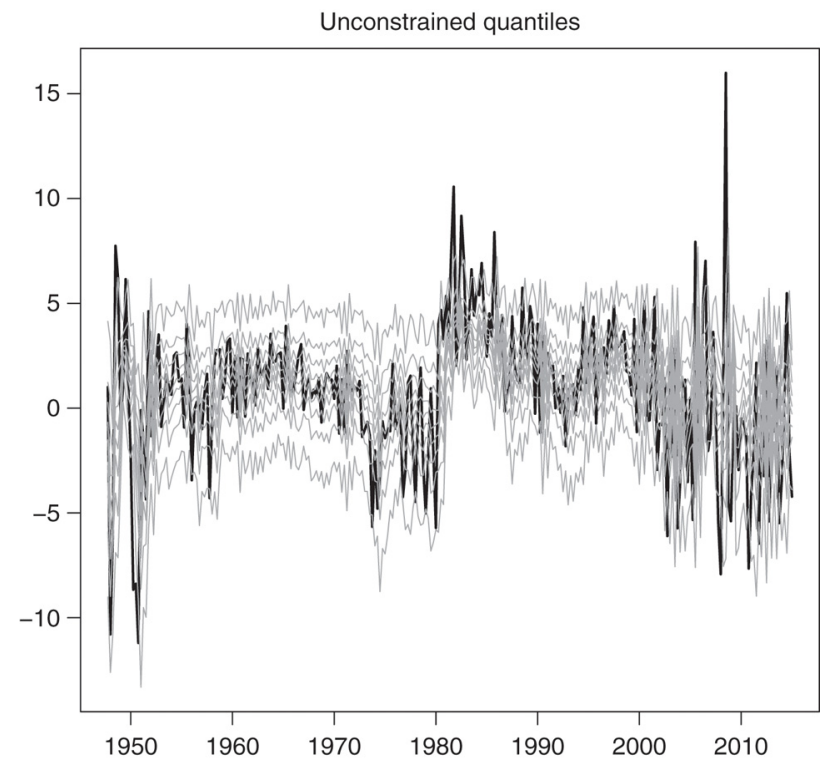

Figure 7: Unconstrained conditional quantiles estimated with the QAR(3) models, specified for $\tau=0.1, \ldots, 0.9$. The thick black line is the real interest rate series, and the light grey lines are the 9 estimated conditional quantiles from $\tau=0.1$ (lowest grey line) to $\tau=0.9$ (highest grey line).

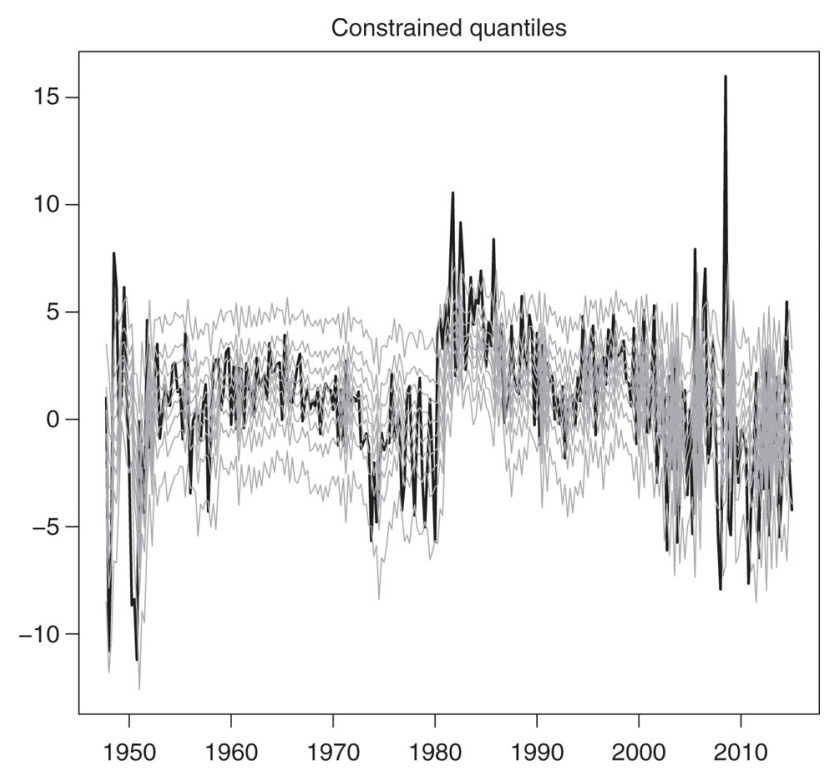

Figure 8: Non-crossing conditional quantiles estimated with the QAR(3) models, specified for $\tau=0.1, \ldots, 0.9$. The thick black line is the real interest rate series, and the light grey lines are the 9 estimated conditional quantiles from $\tau=0.1$ (lowest grey line) to $\tau=0.9$ (highest grey line). 


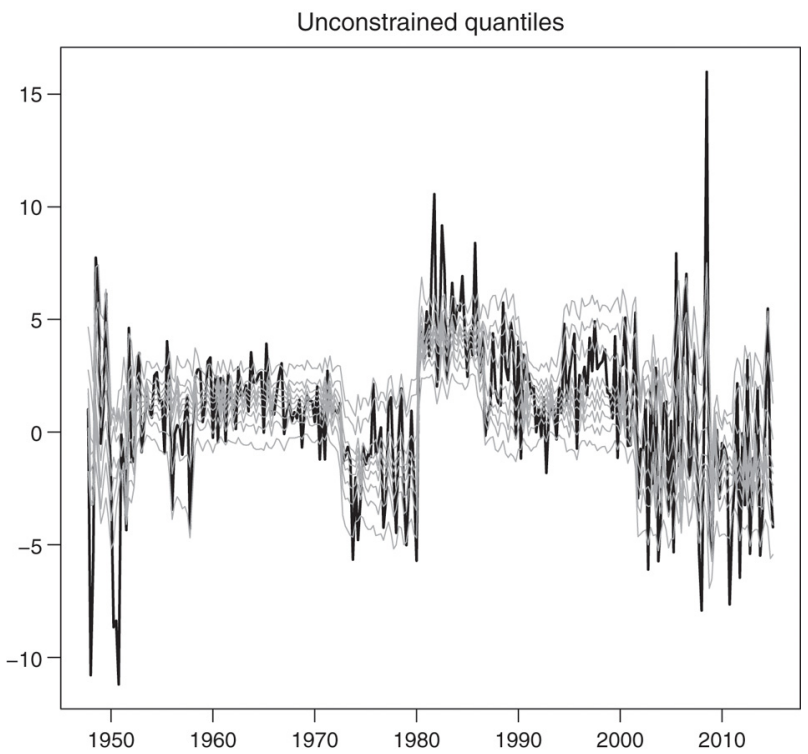

Figure 9: Unconstrained conditional quantiles estimated with the MSQAR(3, 3) models, specified for $\tau=0.1, \ldots, 0.9$. The thick black line is the real interest rate series, and the light grey lines are the 9 estimated conditional quantiles from $\tau=$ 0.1 (lowest grey line) to $\tau=0.9$ (highest grey line).

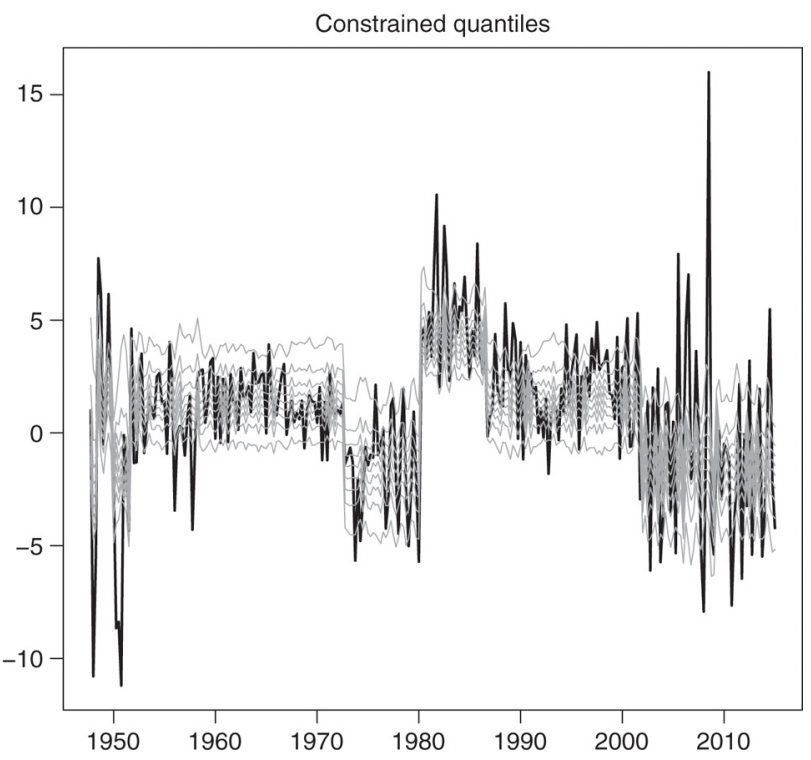

Figure 10: Non-crossing conditional quantiles estimated with the MSQAR $(3,3)$ models, specifed for $\tau=0.1, \ldots, 0.9$. The thick black line is the real interest rate series, and the light grey lines are the 9 estimated conditional quantiles from $\tau=$ 0.1 (lowest grey line) to $\tau=0.9$ (highest grey line).

As Koenker and Xiao (2006), §4 explain, the crossing problem is potentially more acute in QAR models than in ordinary quantile regressions with exogenous covariates, since the support of the regressors is determined within the autoregressive model. So perhaps not surprisingly the estimated quantiles under the non-linear MSQAR specification cross 15 times. Among these, the most notable occurrences in Figure 9 are the 8 crossings between the quantiles at levels 0.7 and 0.8 in 1949Q2, 1949Q3, 1949Q4, 1955Q2, 1981Q3, 1989Q2, 2001Q2, and 2008Q2. Figure 10 shows the refitted MSQAR $(3,3)$ quantiles under the non-crossing restriction. Comparing the MSQAR quantiles in Figure 10 with the QAR quantiles in Figure 8 shows the improvements in terms of fit achieved with the non-linear specification. In line with Garcia and Perron (1996), a Markov-switching model (subject to the non-crossing quantile restriction) appears to better capture the short-term dynamics of the real interest rate.

Another interesting model assessment is a test of correct quantile specification at all quantile levels $\tau=0.1$, $0.2, \ldots, 0.9$, jointly. For this purpose, we use the test procedure of Escanciano and Velasco (2010). Since this test applies to both in-sample predictions and out-of-sample forecasts, we present the outcomes all together in the next section. The results (in Table 11) show the importance of imposing the non-crossing quantile restriction to achieve a correct MSQAR specification. 


\subsection{Out-of-sample forecasting results}

In order to examine the out-of-sample forecasting performance of the MSQAR model, we used a 150-quarter rolling window over the sample period to produce one-quarter ahead forecasts. This results in 123 sets of out-ofsample quantile forecasts at levels $\tau=0.1,0.2, \ldots, 0.9$ from 1984Q3 to 2015Q1. To reduce the computational cost, we kept $\tau_{i^{*}}$ fixed at 0.5 in the procedure for computing the predicted state classifications and the non-crossing predicted quantiles. The quantile forecasts in any given quarter were then made conditional on the prediction of the next quarter's most likely regime.

If we let $Q_{y_{t+1}}(\tau)$ denote the forecast of $y_{t+1}$ 's quantile at level $\tau$, then an ideal forecast would be such that

$$
\operatorname{Pr}\left(y_{t+1} \leq Q_{y_{t+1}}(\tau) \mid \mathfrak{\mho}_{t}\right)=\tau,
$$

where $\mathfrak{F}_{t}$ is the information set available at time $t$. This is the fundamental building block used to devise backtests of value-at-risk (VaR) forecasting models; see Kupiec (1995), Christoffersen (1998), Engle and Manganelli (2004), Escanciano and Velasco (2010), and Gaglianone et al. (2011). Indeed, a VaR corresponds to a conditional quantile of a financial loss distribution. Following the VaR backtesting literature, we first computed the violation rate $\hat{\tau}$, defined as the number of quantile exceedances (violations) divided by the evaluation sample size. As in Gerlach, Chen, and Chan (2011), the quantile forecasting performances can be summarized with the ratios $\hat{\tau} / \tau$ for each model, which ideally should be close to 1 . Otherwise if $\hat{\tau} / \tau<1$, then the conditional quantile is underestimated, while a violation ratio $\hat{\tau} / \tau>1$ means that the conditional quantile is overestimated.

Table 9 reports the out-of-sample quantile violation ratios for the unconstrained and non-crossing versions of the QAR(3) and MSQAR $(3,3)$ models. For each model, the last column reports the average of the violation ratios across all 9 quantile levels $\tau$. In general, we see that imposing the non-crossing restriction improves the quantile forecasts by bringing their violation ratios closer to 1 . Looking at the last column, for instance, we see that the average violation ratio decreases from 1.2 to 1.174 for the QAR(3) model, and from 1.086 to 1.037 for the MSQAR $(3,3)$ model. Among the four specifications, the best one is clearly the MSQAR $(3,3)$ under the non-crossing restriction. This makes clear the value added of restricting the quantile forecasts to not cross one another in addition to allowing for Markov-switching effects. A detailed examination of Table 9 suggests that the QAR model performs well for the middle quantiles $(\tau=0.5,0.6,0.7)$ while the MSQAR offers improvements for the tails of the conditional distribution.

Table 9: Out-of-sample quantile violation ratios.

\begin{tabular}{|c|c|c|c|c|c|c|c|c|c|c|}
\hline & \multicolumn{10}{|c|}{$\tau$} \\
\hline & 0.1 & 0.2 & 0.3 & 0.4 & 0.5 & 0.6 & 0.7 & 0.8 & 0.9 & Average \\
\hline \multicolumn{11}{|c|}{ Panel A: Unconstrained models } \\
\hline QAR(3) & 1.951 & 1.504 & 1.301 & 1.138 & 1.024 & 1.003 & 0.987 & 0.965 & 0.930 & 1.200 \\
\hline $\operatorname{MSQAR}(3,3)$ & 1.382 & 1.239 & 1.165 & 0.996 & 0.959 & 1.016 & 1.034 & 1.026 & 0.958 & 1.086 \\
\hline \multicolumn{11}{|c|}{ Panel B: Constrained models } \\
\hline QAR(3) & 1.951 & 1.382 & 1.219 & 1.097 & 1.024 & 1.016 & 0.964 & 0.965 & 0.949 & 1.174 \\
\hline $\operatorname{MSQAR}(3,3)$ & 1.220 & 0.989 & 1.328 & 1.077 & 0.959 & 0.967 & 0.906 & 0.915 & 0.976 & 1.037 \\
\hline
\end{tabular}

This table reports the ratios $\hat{\tau} / \tau$, where $\hat{\tau}$ is the empirical violation rate observed out of sample and $\tau$ is the nominal quantile level. At each quantile level, the bold entries indicate the model whose ratio is closest to the ideal value of one. The last column reports the average of the ratios across quantile levels.

The forecasting gains were further assessed by testing their statistical significance. Table 10 reports the $p$ values associated with tests of the null hypothesis of a correct quantile specification at level $\tau$. Results are reported for the unconditional coverage (UC) test of Kupiec (1995), the conditional coverage test (CC) of Christoffersen (1998), the dynamic quantile (DQ) test of Engle and Manganelli (2004) using four lags, and the quantile regression-based test for value-at-risk models (VQR) of Gaglianone et al. (2011). These tests are quite standard in the VaR forecast evaluation literature. Looking simply at the number of test outcomes that are significant at the nominal 5\% level (bold entries), we see from Table 10 that the greatest benefits come when moving from the linear QAR model to the non-linear MSQAR model. Indeed there are 19 instances in which the unconstrained QAR model is rejected, while there are only 2 such instances for the unconstrained MSQAR model.

Table 10: Marginal quantile specification tests: out-of-sample results.

$\tau=0.1 \quad \tau=0.2$

$\tau=0.3$ 


\begin{tabular}{|c|c|c|c|c|c|c|c|c|c|c|c|c|}
\hline & UC & $\mathrm{CC}$ & DQ & VQR & UC & $\mathrm{CC}$ & DQ & VQR & UC & $\mathrm{CC}$ & DQ & VQR \\
\hline \multicolumn{13}{|c|}{ Panel A: Unconstrained models } \\
\hline QAR(3) & 0.00 & 0.01 & 0.39 & 0.02 & 0.01 & 0.02 & 0.70 & 0.02 & 0.03 & 0.02 & 0.67 & 0.02 \\
\hline $\operatorname{MSQAR}(3,3)$ & 0.18 & 0.20 & 0.87 & 0.68 & 0.82 & 0.81 & 0.22 & 0.93 & 0.24 & 0.14 & 0.45 & 0.33 \\
\hline \multicolumn{13}{|c|}{ Panel B: Constrained models } \\
\hline QAR(3) & 0.00 & 0.00 & 0.43 & 0.00 & 0.04 & 0.06 & 0.17 & 0.11 & 0.12 & 0.03 & 0.21 & 0.27 \\
\hline \multirow[t]{3}{*}{ MSQAR $(3,3)$} & 0.43 & 0.70 & 0.15 & 0.30 & 0.33 & 0.20 & 0.77 & 0.59 & 0.24 & 0.11 & 0.40 & 0.33 \\
\hline & & & & $\tau=0.4$ & & & & $\tau=0.5$ & & & & $\tau=0.6$ \\
\hline & $\mathrm{UC}$ & $\mathrm{CC}$ & DQ & VQR & UC & $\mathrm{CC}$ & DQ & VQR & UC & $\mathrm{CC}$ & DQ & VQR \\
\hline \multicolumn{13}{|c|}{ Panel C: Unconstrained models } \\
\hline QAR(3) & 0.21 & 0.01 & 0.77 & 0.29 & 0.79 & 0.00 & 0.91 & 0.12 & 0.97 & 0.01 & 0.93 & 0.00 \\
\hline $\operatorname{MSQAR}(3,3)$ & 0.97 & 0.27 & 0.68 & 0.37 & 0.65 & 0.17 & 0.96 & 0.03 & 0.82 & 0.60 & 0.35 & 0.03 \\
\hline \multicolumn{13}{|c|}{ Panel D: Constrained models } \\
\hline QAR(3) & 0.38 & 0.14 & 0.17 & 0.72 & 0.79 & 0.00 & 0.56 & 0.12 & 0.82 & 0.01 & 0.16 & 0.00 \\
\hline \multirow[t]{3}{*}{$\operatorname{MSQAR}(3,3)$} & 0.49 & 0.01 & 0.78 & 0.05 & 0.65 & 0.17 & 0.21 & 0.03 & 0.29 & 0.09 & 0.11 & 0.20 \\
\hline & & & & $\tau=0.7$ & & & & $\tau=0.8$ & & & & $\tau=0.9$ \\
\hline & $\mathrm{UC}$ & $\mathrm{CC}$ & DQ & VQR & UC & $\mathrm{CC}$ & DQ & VQR & UC & $\mathrm{CC}$ & DQ & VQR \\
\hline \multicolumn{13}{|c|}{ Panel E: Unconstrained models } \\
\hline QAR(3) & 0.83 & 0.01 & 0.17 & 0.01 & 0.45 & 0.05 & 0.90 & 0.00 & 0.03 & 0.12 & 0.18 & 0.01 \\
\hline $\operatorname{MSQAR}(3,3)$ & 0.57 & 0.25 & 0.47 & 0.22 & 0.55 & 0.65 & 0.83 & 0.15 & 0.18 & 0.24 & 0.83 & 0.23 \\
\hline \multicolumn{13}{|c|}{ Panel F: Constrained models } \\
\hline QAR(3) & 0.54 & 0.04 & 0.37 & 0.01 & 0.45 & 0.04 & 0.27 & 0.00 & 0.11 & 0.18 & 0.19 & 0.01 \\
\hline MSQAR(3, 3) & 0.23 & 0.11 & 0.14 & 0.13 & 0.71 & 0.47 & 0.25 & 0.32 & 0.43 & 0.54 & 0.90 & 0.10 \\
\hline
\end{tabular}

This table reports the $p$-values associated with tests of a correct quantile specification at level $\tau$. Results are reported for the unconditional coverage (UC) test of Kupiec (1995), the conditional coverage test (CC) of Christoffersen (1998), the dynamic quantile (DQ) test of Engle and Manganelli (2004), and the quantile regression-based test for value-at-risk models (VQR) of Gaglianone et al. (2011). Values < 0.01 are reported as zero and bold face entries indicate statistical significance at the nominal $5 \%$ level.

Table 11 reports the $p$-values associated with three versions of the Escanciano and Velasco (2010) test for correct quantile specification at all quantile levels: $C v M_{T}$ is based on the Cramér-von Mises functional, $K_{T}$ is an extended version of the Kupiec (1995) statistic, and $C_{T}$ is an extended version of the Christoffersen (1998) statistic. These tests are computed according to Eqs. (10), (11), and (13) in Escanciano and Velasco (2010), respectively, with the $m=9$ equi-distributed points $\tau=0.1,0.2, \ldots, 0.9$ and $b=150$ for their subsampling procedure. The key takeaway from Table 11 is that the $\operatorname{MSQAR}(3,3)$ subject to the non-crossing restriction is the only model that passes the correct specification tests, both in and out of sample.

Table 11: Joint quantile specification tests.

\begin{tabular}{|c|c|c|c|c|c|c|}
\hline & \multicolumn{3}{|c|}{ In-sample results } & \multicolumn{3}{|c|}{ Out-of-sample results } \\
\hline & $C v M_{T}$ & $K_{T}$ & $C_{T}$ & $C v M_{T}$ & $K_{T}$ & $C_{T}$ \\
\hline \multicolumn{7}{|c|}{ Panel A: Unconstrained models } \\
\hline QAR(3) & 0.041 & 0.187 & 0.073 & 0.000 & 0.000 & 0.000 \\
\hline $\operatorname{MSQAR}(3,3)$ & 0.911 & 0.919 & 0.106 & 0.000 & 0.000 & 0.001 \\
\hline \multicolumn{7}{|c|}{ Panel B: Constrained models } \\
\hline QAR(3) & 0.341 & 0.000 & 0.122 & 0.000 & 0.000 & 0.000 \\
\hline $\operatorname{MSQAR}(3,3)$ & 0.740 & 0.772 & 0.642 & 0.382 & 0.244 & 0.069 \\
\hline
\end{tabular}

This table reports the $p$-values associated with the Escanciano and Velasco (2010) test of a correct quantile specification at levels $\tau=0.1$, $0.2, \ldots, 0.9$, jointly. In-sample and out-of-sample results are reported using three different versions of the test: $C v M_{T}$ is based on the Cramér-von Mises functional, $K_{T}$ is an extended version of the Kupiec (1995) statistic, and $C_{T}$ is an extended version of the Christoffersen (1998) statistic. These tests are computed according to Eqs. (10), (11), and (13) in Escanciano and Velasco (2010), respectively. Values < 0.001 are reported here as zero and bold face entries indicate statistical significance at the nominal $5 \%$ level.

\section{Conclusion}

We have extended the class of linear quantile autoregression models of Koenker and Xiao (2006) by allowing for the possibility of Markov-switching regime changes à la Hamilton (1989) in the conditional distribution of the response variable. We proposed a complete Bayesian methodology for: (i) estimation and inference; (ii) 
specification analysis of the number of regimes and the number of autoregressive lags; and (iii) enforcing the quantile monotonicity restriction that must be satisfied for any distribution to be well defined.

The Bayesian calculations are easily implemented, since all complete conditional densities used in the Gibbs sampler have closed-form expressions. As in Gelfand, Smith, and Lee (1992), Gibbs sampling is the key building block for the proposed stepwise re-estimation procedure that ensures non-crossing quantiles. Monte Carlo experiments and an illustrative empirical application show how much stronger inference and forecasting can be when the quantile monotonicity restriction is imposed.

\section{Appendices}

\section{A Filtering and MSQAR likelihood}

The likelihood function of the MSQAR model is obtained as a byproduct of the basic filter algorithm developed by Hamilton (1989) to draw probabilistic inferences about the unobserved states $s_{t-p+1}, \ldots, s_{t}$ given observations on $y_{t}$. The filter is initialized with $p\left(\mathbf{s}_{1: p} \mid \mathbf{y}_{1: p} ; \boldsymbol{\theta}(\tau), \delta, \tau\right)$ and then iterates on the following steps for $t=p+1, \ldots$, $T$ :

Step 1. Given $p\left(\mathbf{s}_{t-p: t-1} \mid \mathbf{y}_{1: t-1} ; \boldsymbol{\theta}(\tau), \delta, \tau\right)$, compute

$$
p\left(\mathbf{s}_{t-p: t} \mid \mathbf{y}_{1: t-1} ; \boldsymbol{\theta}(\tau), \delta, \tau\right)=p\left(s_{t} \mid s_{t-1} ; \tau\right) \times p\left(\mathbf{s}_{t-p: t-1} \mid \mathbf{y}_{1: t-1} ; \boldsymbol{\theta}(\tau), \delta, \tau\right),
$$

where $p\left(s_{t} \mid s_{t-1} ; \tau\right)$ refers to the transition probabilities in (8)

Step 2. Compute the filtered probability as

$$
p\left(\mathbf{s}_{t-p+1: t} \mid \mathbf{y}_{1: t} ; \boldsymbol{\theta}(\tau), \delta, \tau\right)=\sum_{s_{t-p}} p\left(\mathbf{s}_{t-p: t} \mid \mathbf{y}_{1: t} ; \boldsymbol{\theta}(\tau), \delta, \tau\right),
$$

where

$$
p\left(\mathbf{s}_{t-p: t} \mid \mathbf{y}_{1: t} ; \boldsymbol{\theta}(\tau), \delta, \tau\right)=\frac{f\left(y_{t}, \mathbf{s}_{t-p: t} \mid \mathbf{y}_{1: t-1} ; \boldsymbol{\theta}(\tau), \delta, \tau\right)}{f\left(y_{t} \mid \mathbf{y}_{1: t-1} ; \boldsymbol{\theta}(\tau), \delta, \tau\right)} .
$$

The likelihood of $y_{t}$ appearing in the denominator of this last expression is given by

$$
f\left(y_{t} \mid \mathbf{y}_{1: t-1} ; \boldsymbol{\theta}(\tau), \delta, \tau\right)=\sum_{s_{t}} \ldots \sum_{s_{t-p}} f\left(y_{t}, \mathbf{s}_{t-p: t} \mid \mathbf{y}_{1: t-1} ; \boldsymbol{\theta}(\tau), \delta, \tau\right),
$$

with

$$
f\left(y_{t}, \mathbf{s}_{t-p: t} \mid \mathbf{y}_{1: t-1} ; \boldsymbol{\theta}(\tau), \delta, \tau\right)=f\left(y_{t} \mid \mathbf{y}_{1: t-1}, \mathbf{s}_{t-p: t} ; \boldsymbol{\theta}(\tau), \delta, \tau\right) \times p\left(\mathbf{s}_{t-p: t} \mid \mathbf{y}_{1: t-1} ; \boldsymbol{\theta}(\tau), \delta, \tau\right),
$$

and where $f\left(y_{t} \mid \mathbf{y}_{1: t-1}, \mathbf{s}_{t-p: t} ; \boldsymbol{\theta}(\tau), \delta, \tau\right)$ is defined in (10).

As a byproduct of these filtering steps, the sample MSQAR likelihood function could be obtained according to

$$
f\left(\mathbf{y}_{p+1: T} \mid \mathbf{y}_{1: p} ; \boldsymbol{\theta}(\tau), \delta, \tau\right)=\prod_{t=p+1}^{T} f\left(y_{t} \mid \mathbf{y}_{1: t-1} ; \boldsymbol{\theta}(\tau), \delta, \tau\right),
$$

and, as Hamilton (1989) explains, rather than using $p\left(\mathbf{s}_{1: p} \mid \mathbf{y}_{1: p} ; \boldsymbol{\theta}(\tau), \delta, \tau\right)$ it is easier to start up the filter with $p\left(\mathbf{s}_{1: p} \mid \mathbf{p}(\tau), \tau\right)$. To compute this unconditional probability, we start with $p\left(s_{1} \mid \tau\right)$ in Assumption 4 and then calculate

$$
p\left(\mathbf{s}_{1: t} \mid \mathbf{p}(\tau), \tau\right)=p\left(s_{t} \mid s_{t-1} ; \tau\right) \times p\left(\mathbf{s}_{1: t-1} ; \tau\right),
$$

recursively for $t=2, \ldots, p$. 


\section{B Gibbs steps}

In the following we shall simplify the notation and use, for example, $\theta(\tau)_{-\mu}$ to denote all the parameters in $\boldsymbol{\theta}(\tau)$ excluding $\boldsymbol{\mu}(\tau)$.

\section{B.1 Sampling the state variables}

In this section we describe two ways to generate draws from the distribution of $\mathbf{s}$ conditional upon $\boldsymbol{\theta}(\tau)$ and y. Specifically, the two methods are single- and multi-move sampling which differ in their computational cost and efficiency. The presentation here closely follows Kim and Nelson (1999), Ch. 9.

\section{B.1.1 Single-move sampling}

The single-move sampler proposed by Albert and Chib (1993) generates samples of $\mathbf{s}$ by drawing $s_{t}$ for each $t$ one by one from each of the following $T$ conditional distributions:

$$
p\left(s_{t} \mid \mathbf{s}_{-t}, \boldsymbol{\theta}(\tau), \mathbf{y}\right),
$$

defined for $t=1, \ldots, T$, where $\mathbf{s}_{-t}=\left\{s_{t_{1}}: 1 \leq t_{1} \leq T, t_{1} \neq t\right\}$.

The key result obtained from Bayes' theorem for single-move sampling is:

$$
p\left(s_{t} \mid \mathbf{s}_{-t}, \boldsymbol{\theta}(\tau), \mathbf{y}\right) \propto p\left(s_{t} \mid s_{t-1} ; \mathbf{p}(\tau), \tau\right) p\left(s_{t+1} \mid s_{t} ; \mathbf{p}(\tau), \tau\right) \prod_{k=t}^{t+p} f\left(y_{k} \mid \mathbf{y}_{1: k-1}, \mathbf{s}_{k-p: k} ; \boldsymbol{\theta}(\tau), \delta, \tau\right)
$$

for $p+1 \leq t \leq T-p+1$. Just as the Hamilton (1989) filter is started up by considering the Markov chain in isolation, we can generate the first $p$ values of $s_{t}$ by first obtaining a draw of $s_{1}$ according to the unconditional probabilities $p\left(s_{1} \mid \tau\right)$ in Assumption 4 and then drawing the next $p-1$ values of $s_{t}$ according to the probabilities:

$$
p\left(s_{t} \mid \mathbf{s}_{-t}, \boldsymbol{\theta}(\tau), \mathbf{y}\right) \propto p\left(s_{t} \mid s_{t-1} ; \mathbf{p}(\tau), \tau\right) p\left(s_{t+1} \mid s_{t} ; \mathbf{p}(\tau), \tau\right),
$$

for $t=2, \ldots, p$. The result in (18) also needs to be slightly modified to deal with the end points. For $t=T-p, \ldots$, $T-1$, the draws of $s_{t}$ are generated with probabilities:

$$
p\left(s_{t} \mid \mathbf{s}_{-t}, \boldsymbol{\theta}(\tau), \mathbf{y}\right) \propto p\left(s_{t} \mid s_{t-1} ; \mathbf{p}(\tau), \tau\right) p\left(s_{t+1} \mid s_{t} ; \mathbf{p}(\tau), \tau\right) \prod_{k=t}^{T} f\left(y_{k} \mid \mathbf{y}_{1: k-1}, \mathbf{s}_{k-p: k} ; \boldsymbol{\theta}(\tau), \delta, \tau\right),
$$

and, when $t=T$, we use

$$
p\left(s_{T} \mid \mathbf{s}_{-T}, \boldsymbol{\theta}(\tau), \mathbf{y}\right) \propto p\left(s_{T} \mid s_{T-1} ; \mathbf{p}(\tau), \tau\right) f\left(y_{T} \mid \mathbf{y}_{1: T-1}, \mathbf{s}_{T-p: T} ; \boldsymbol{\theta}(\tau), \delta, \tau\right) .
$$

With the results in (18)-(21), the normalized probabilities can be calculated as

$$
p\left(s_{t} \mid \mathbf{s}_{-t}, \boldsymbol{\theta}(\tau), \mathbf{y}\right)=\frac{p\left(s_{t} \mid \mathbf{s}_{-t}, \boldsymbol{\theta}(\tau), \mathbf{y}\right)}{\sum_{s_{t}} p\left(s_{t} \mid \mathbf{s}_{-t}, \boldsymbol{\theta}(\tau), \mathbf{y}\right)}
$$

and then drawing $s_{t}$ is just like sampling from a multinomial distribution. Note that the state variable $s_{t}$ is sampled iteratively for each $t=1, \ldots, T$ from these discrete distributions while conditioning on the most recent draw for all other states, $\mathbf{s}_{-t}$. 


\section{B.1.2 Multi-move sampling}

An alternative to the single-move sampler is the multi-move sampling approach of Carter and Kohn (1994), which draws the entire sequence $\mathbf{s}$ from the conditional posterior $p(\mathbf{s} \mid \boldsymbol{\theta}(\tau), \delta, \tau, \mathbf{y})$. As Frühwirth-Schnatter (2006), Ch. 11 describes, the multi-move sampling approach is based on expressing the joint posterior $p(\mathbf{s} \mid \boldsymbol{\theta}(\tau), \delta, \tau, \mathbf{y})$ as

$$
p(\mathbf{s} \mid \boldsymbol{\theta}(\tau), \delta, \tau, \mathbf{y})=p\left(s_{T} \mid \boldsymbol{\theta}(\tau), \delta, \tau, \mathbf{y}\right) \prod_{t=1}^{T-1} p\left(\mathbf{s}_{t} \mid \mathbf{s}_{t+1: T} ; \mathbf{y} ; \boldsymbol{\theta}(\tau), \delta, \tau\right),
$$

where $p\left(s_{T} \mid \boldsymbol{\theta}(\tau), \delta, \tau, \mathbf{y}\right)$ is the filtered probability distribution at time $T$. So given values for $\boldsymbol{\theta}(\tau), \delta$, and the observations $\mathbf{y}$, a multi-move sample of $\mathbf{s}$ is generated according to the following steps:

Step 1. Run the Hamilton (1989) filter described in Appendix A to get $p\left(\mathbf{s}_{t} \mid \boldsymbol{\theta}(\tau), \delta, \tau, \mathbf{y}_{1: t}\right)$, for $t=1, \ldots, T$.

Step 2. Sample $s_{T}$ according to $p\left(s_{T} \mid \boldsymbol{\theta}(\tau), \delta, \tau, \mathbf{y}\right)$, and then, for $t=T-1, T-2, \ldots, 1$, sample $s_{t}$ according to

$$
p\left(s_{t} \mid s_{t+1}, \boldsymbol{\theta}(\tau), \delta, \tau, \mathbf{y}\right)=\frac{p\left(s_{t+1} \mid s_{t}, \mathbf{p}(\tau), \tau\right) p\left(\mathbf{s}_{t} \mid \boldsymbol{\theta}(\tau), \delta, \tau, \mathbf{y}_{1: t}\right)}{\sum_{s_{t}} p\left(s_{t+1} \mid s_{t}, \mathbf{p}(\tau), \tau\right) p\left(\mathbf{s}_{t} \mid \boldsymbol{\theta}(\tau), \delta, \tau, \mathbf{y}_{1: t}\right)}
$$

which, as in the case of the single-move sampler, amounts to sampling a multinomial distribution.

A computational disadvantage of this approach is that it requires a run of the filtering algorithm each time a sample of state variables is generated. On the other hand, multi-move sampling may have better mixing properties than the sampler that generates the states one at a time in the case of highly correlated Markov chains (Albert \& Chib, 1993; McCulloch \& Tsay, 1994; Scott, 2002). In Section 5, we examine this issue in the context of the proposed MSQAR model.

\section{B.2 Sampling the transition probabilities}

Observe that upon conditioning on the sequence of state variables $\mathbf{s}$, the posterior distribution of transition probabilities $p_{i j}(\tau)$ becomes independent of $\mathbf{y}$ and all the other model parameters. Let $\mathbf{p}_{i} .(\tau)$ denote the $i$ th row of the transition probability matrix $\mathbf{P}(\tau)$. We specify the prior distribution for $\mathbf{p}_{i} .(\tau)$ as a Dirichlet distribution, $D\left(\alpha_{i 1}, \ldots, \alpha_{i K}\right)$. Since the Dirichlet distribution is the conjugate prior for the multinomial distribution, the posterior distributions are also Dirichlet distributions:

$$
p\left(\mathbf{p}_{i .}(\tau) \mid \mathbf{s}\right) \sim D\left(\alpha_{i 1}+\mathbb{N}_{i 1}[\mathbf{s}], \ldots, \alpha_{i K}+\mathbb{N}_{i K}[\mathbf{s}]\right),
$$

where $\mathbb{N}_{i j}[\mathbf{s}]$ counts the number of transitions from $i$ to $j$ occurring in $\mathbf{s}$, as before.

\section{B.3 Sampling $\mu(\tau)$ and $\phi(\tau)$}

Once the states have been simulated, the model can be considerably simplified by expressing it as a linear function of the parameters. Indeed, given values of $\mathbf{s}$, the model in (12) can be transformed into

$$
y_{t}^{*}=\sum_{i=1}^{K} \mu_{i}(\tau) s_{i, t}^{*}+\gamma v_{t}+\xi \sqrt{\delta v_{t}} z_{t}
$$

where $y_{t}^{*}=y_{t}-\sum_{j=1}^{p} \phi_{j}(\tau) y_{t-j}$ and $s_{i, t}^{*}=\llbracket\left[s_{t}=i\right]-\sum_{j=1}^{p} \phi_{j}(\tau) \llbracket\left[s_{t-j}=i\right]$, for $i=1, \ldots, K$. In matrix notation, this becomes

$$
\mathbf{y}^{*}=\mathbf{S}^{*} \boldsymbol{\mu}(\tau)+\gamma \mathbf{v}+\xi \sqrt{\delta \mathbf{v}} \mathbf{z},
$$

where $\mathbf{y}^{*}=\left[y_{p+1}^{*}, \ldots, y_{T}^{*}\right]^{\prime}$ and $\mathbf{v}=\mathbf{v}_{p+1: T}=\left[v_{p+1}, \ldots, v_{T}\right]^{\prime}$ are column vectors with $T-p$ rows, and $\mathbf{S}^{*}=$ $\left[\mathbf{s}_{1, p+1: T}^{*}, \ldots, \mathbf{s}_{K, p+1: T}^{*}\right]$ is a $(T-p) \times K$ matrix. Following standard practice, we assume that

$$
\boldsymbol{\mu}(\tau) \sim N\left(\boldsymbol{\mu}_{0}(\tau), \boldsymbol{\Sigma}_{\mu, 0}(\tau)\right)\left[\left[\mu_{1}(\tau)<\mu_{2}(\tau)<\ldots<\mu_{K}(\tau)\right]\right.
$$


where $\boldsymbol{\mu}_{0}(\tau)$ and $\boldsymbol{\Sigma}_{\mu, 0}(\tau)$ comprise the known hyperparameters of this prior distribution. The usual Bayesian calculation then yields the posterior distribution:

$$
\boldsymbol{\mu}(\tau) \mid \boldsymbol{\theta}(\tau)_{-\mu}, \mathbf{s}, \mathbf{v}, \mathbf{y} \sim N\left(\boldsymbol{\mu}_{1}(\tau), \boldsymbol{\Sigma}_{\mu, 1}(\tau)\right) \square\left[\mu_{1}(\tau)<\mu_{2}(\tau)<\cdots<\mu_{K}(\tau)\right],
$$

where $\boldsymbol{\Sigma}_{\mu, 1}(\tau)=\left(\boldsymbol{\Sigma}_{\mu, 0}(\tau)^{-1}+\mathbf{S}^{* \prime} \mathbf{S}^{*}\right)^{-1}$ and $\boldsymbol{\mu}_{1}(\tau)=\boldsymbol{\Sigma}_{\mu, 1}(\tau)\left(\boldsymbol{\Sigma}_{\mu, 0}(\tau)^{-1} \boldsymbol{\mu}_{0}(\tau)+\mathbf{S}^{* \prime} \mathbf{y}^{*}\right)$. Observe that the identification constraint in Assumption 1 is imposed here using "rejection sampling."

If we now let $y_{t}^{* *}=y_{t}-\mu\left(\tau, s_{t}\right)$, then model (12) can be written in matrix form as

$$
\mathbf{y}^{* *}=\mathbf{X} \boldsymbol{\phi}(\tau)+\gamma \mathbf{v}+\xi \sqrt{\delta \mathbf{v}} \mathbf{z}
$$

where $\mathbf{y}^{* *}=\left(y_{p+1}^{* *}, \ldots, y_{T}^{* *}\right)^{\prime}$ and $\mathbf{X}=\left[\mathbf{y}_{p: T-1}^{* *}, \ldots, \mathbf{y}_{1: T-p}^{* *}\right]$. As before, we use the natural conjugate normal prior for linear models and assume that

$$
\boldsymbol{\phi}(\tau) \sim N\left(\phi_{0}(\tau), \boldsymbol{\Sigma}_{\phi, 0}(\tau)\right) \mathbb{S}[\boldsymbol{\phi}(\tau)],
$$

where $\mathbb{S}[\phi(\tau)]$ is an indicator function which equals one when the roots of $\phi(\tau, L)=0$ lie outside the unit circle, and zero otherwise. The posterior is then found to be

$$
\boldsymbol{\phi}(\tau) \mid \boldsymbol{\theta}(\tau)_{-\phi}, \mathbf{s}, \mathbf{v}, \mathbf{y} \sim N\left(\phi_{1}(\tau), \boldsymbol{\Sigma}_{\phi, 1}(\tau)\right) \mathbb{S}[\boldsymbol{\phi}(\tau)],
$$

with $\boldsymbol{\Sigma}_{\phi, 1}(\tau)=\left(\boldsymbol{\Sigma}_{\phi, 0}(\tau)^{-1}+\mathbf{X}^{\prime} \mathbf{X}\right)^{-1}$ and $\boldsymbol{\phi}_{1}(\tau)=\boldsymbol{\Sigma}_{\phi, 1}(\tau)\left(\boldsymbol{\Sigma}_{\phi, 0}(\tau)^{-1} \boldsymbol{\phi}_{0}(\tau)+\mathbf{X}^{\prime} \mathbf{y}^{* *}\right)$. Here again we use rejection sampling so that only the draws from the posterior distribution satisfying the stationarity constraint (Assumption 2) are retained.

\section{B.4 Sampling $v$ and $\delta$}

From (12) we see that given $\mathbf{s}, \boldsymbol{\theta}(\tau)$, and $\mathbf{v}$, the conditional distribution of $y_{t}$ is normal with mean $\mu\left(\tau, s_{t}\right)+$ $\sum_{j=1}^{p} \phi_{j}(\tau)\left(y_{t-j}-\mu\left(\tau, s_{t-j}\right)\right)+\gamma v_{t}$ and variance $\xi^{2} \delta v_{t}$ so that

$$
f\left(y_{t} \mid \mathbf{s}, \boldsymbol{\theta}(\tau), \mathbf{v}, \mathbf{y}\right) \propto\left(\delta v_{t}\right)^{1 / 2} \exp \left\{-\frac{\left(y_{t}-\ell_{t}-\gamma v_{t}\right)^{2}}{2 \xi^{2} \delta v_{t}}\right\},
$$

with $\ell_{t}=\mu\left(\tau, s_{t}\right)+\sum_{j=1}^{p} \phi_{j}(\tau)\left(y_{t-j}-\mu\left(\tau, s_{t-j}\right)\right)$ denoting the conditional location. Upon noticing that $v_{t} \sim \mathscr{E}(\delta)$, we find as in Kozumi and Kobayashi (2011) that the full conditional distribution of $v_{t}$ given $\mathbf{y}$, s, and $\boldsymbol{\theta}(\tau)$ is proportional to

$$
v_{t}^{-1 / 2} \exp \left\{-\frac{1}{2}\left(\chi_{t}^{2} v_{t}^{-1}+\psi_{t}^{2} v_{t}\right)\right\}, \text { for } t=p+1, \ldots, T,
$$

where $\chi_{t}^{2}=\left(y_{t}-\ell_{t}\right)^{2} /\left(\xi^{2} \delta\right)$ and $\psi_{t}^{2}=2 / \delta+\gamma^{2} /\left(\xi^{2} \delta\right)$. Expression (22) is recognized as the kernel of a generalized inverse Gaussian distribution so that

$$
v_{t} \mid \mathbf{y}, \mathbf{s}, \boldsymbol{\theta}(\tau) \sim \operatorname{GIG}\left(1 / 2, \chi_{t}, \psi_{t}\right), \text { for } t=p+1, \ldots, T,
$$

where the $\operatorname{GIG}(\nu, a, b)$ density is given by

$$
f(x \mid v, a, b)=\frac{(b / a)^{v}}{2 K_{v}(a b)} x^{\nu-1} \exp \left\{\frac{1}{2}\left(a^{2} x^{-1}+b^{2} x\right)\right\}, \quad x>0,-\infty<v<\infty, a, b \geq 0,
$$

with $K_{v}(\cdot)$ denoting a modified Bessel function of the third kind with index $v$; see Dagpunar (1989) for more details and an efficient algorithm to simulate from the GIG distribution.

For the scale parameter $\delta$ we assume the usual inverse Gamma prior distribution with parameters $c_{0} / 2$ and $d_{0} / 2$, representing this here as $\delta \sim \operatorname{IG}\left(c_{0} / 2, d_{0} / 2\right)$. Since the joint conditional distribution of $y_{t}$ and $v_{t}$ is given as 
the product of the normal distribution (with mean $\ell_{t}+\gamma v_{t}$ and variance $\xi^{2} \delta v_{t}$ ) and the exponential distribution (with parameter $\delta$ ), the posterior distribution of $\delta$ is propositional to

$$
\left(\frac{1}{\delta}\right)^{c_{1} / 2+1+3(T-p) / 2} \exp \left\{-\frac{1}{\delta}\left(\frac{d_{0}}{2}+\sum_{t=p+1}^{T} v_{t}+\sum_{t=p+1}^{T} \frac{\left(y_{t}-\ell_{t}-\gamma v_{t}\right)^{2}}{2 \xi^{2} v_{t}}\right)\right\} .
$$

This expression is recognized as the kernel of the inverse Gamma distribution, meaning that

$$
\delta \mid \mathbf{y}, \mathbf{s}, \mathbf{v}, \boldsymbol{\theta}(\tau)_{-\delta} \sim \mathrm{IG}\left(c_{1} / 2, d_{1} / 2\right),
$$

with $c_{1}=c_{0}+3(T-p)$ and $d_{1}=d_{0}+2 \sum_{t=p+1}^{T} v_{t}+\sum_{t=p+1}^{T}\left(y_{t}-\ell_{t}-\gamma v_{t}\right)^{2} / \xi^{2} v_{t}$.

\section{Computation of the marginal likelihood}

The first term on the right-hand side of (13) is the log of the MSQAR likelihood function in (5) evaluated at $\boldsymbol{\theta}(\tau)^{*}$. The second term on the right-hand side of (13) is the log of the prior density at $\boldsymbol{\theta}(\tau)^{*}$. This term can be written as

$$
\begin{aligned}
\log \pi\left(\boldsymbol{\theta}(\tau)^{*}\right) & =\log f_{N}\left(\boldsymbol{\mu}(\tau)^{*} ; \boldsymbol{\mu}_{0}(\tau), \boldsymbol{\Sigma}_{\mu, 0}(\tau)\right)+\log f_{N}\left(\boldsymbol{\phi}(\tau)^{*} ; \boldsymbol{\phi}_{0}(\tau), \boldsymbol{\Sigma}_{\phi, 0}(\tau)\right) \\
& +\log f_{I G}\left(\delta^{*} ; c_{0} / 2, d_{0} / 2\right)+\sum_{i=1}^{K} \log f_{D}\left(\mathbf{p}_{i .}(\tau)^{*} ; \alpha_{i 1}, \ldots, \alpha_{i K}\right),
\end{aligned}
$$

where $f_{N}$ is the multivariate normal density, $f_{I G}$ is the inverted Gamma density, and $f_{D}$ is the Dirichlet density.

The log of the posterior ordinate estimate appearing as the third term on the right-hand side of (13) requires further computations. We begin with the decomposition

$$
\begin{aligned}
\log \pi\left(\boldsymbol{\theta}(\tau)^{*} \mid \mathbf{y}\right) & =\log \pi\left(\boldsymbol{\mu}(\tau)^{*} \mid \mathbf{y}\right)+\log \pi\left(\boldsymbol{\phi}(\tau)^{*} \mid \mathbf{y}, \boldsymbol{\mu}(\tau)^{*}\right) \\
& +\log \pi\left(\delta(\tau)^{*} \mid \mathbf{y}, \boldsymbol{\mu}(\tau)^{*}, \boldsymbol{\phi}(\tau)^{*}\right)+\sum_{i=1}^{K} \log \pi\left(\mathbf{p}_{i}(\tau)^{*} \mid \mathbf{y}, \boldsymbol{\mu}(\tau)^{*}, \boldsymbol{\phi}(\tau)^{*}, \delta(\tau)^{*}\right),
\end{aligned}
$$

which is obtained by first writing the joint posterior as a product of conditional posteriors. The ordinate $\pi\left(\boldsymbol{\mu}(\tau)^{*} \mid \mathbf{y}\right)$ can be expressed as

$$
\pi\left(\boldsymbol{\mu}(\tau)^{*} \mid \mathbf{y}\right)=\int \pi\left(\boldsymbol{\mu}(\tau)^{*} \mid \mathbf{y}, \boldsymbol{\phi}(\tau), \delta, \mathbf{P}(\tau), \mathbf{s}, \mathbf{v}\right) \pi(\boldsymbol{\phi}(\tau), \delta, \mathbf{P}(\tau), \mathbf{s}, \mathbf{v} \mid \mathbf{y}) d \boldsymbol{\phi}(\tau) d \delta d \mathbf{P}(\tau) d \mathbf{s} d \mathbf{v},
$$

which can then be estimated from the output of the Gibbs algorithm as

$$
\hat{\pi}\left(\boldsymbol{\mu}(\tau)^{*} \mid \mathbf{y}\right)=N^{-1} \sum_{n=1}^{N} \pi\left(\boldsymbol{\mu}(\tau)^{*} \mid \mathbf{y}, \boldsymbol{\phi}(\tau)^{n}, \delta^{n}, \mathbf{P}(\tau)^{n}, \mathbf{s}^{n}, \mathbf{v}^{n}\right),
$$

since $\boldsymbol{\phi}(\tau)^{n}, \delta^{n}, \mathbf{P}(\tau)^{n}, \mathbf{s}^{n}, \mathbf{v}^{n}$ is a draw from the conditional distribution of $\boldsymbol{\phi}(\tau), \delta, \mathbf{P}(\tau), \mathbf{s}, \mathbf{v}$ given $\mathbf{y}$

We next turn to the estimation of the ordinate $\pi\left(\boldsymbol{\phi}(\tau)^{*} \mid \mathbf{y}, \boldsymbol{\mu}(\tau)^{*}\right)$, expressed as

$$
\pi\left(\boldsymbol{\phi}(\tau)^{*} \mid \mathbf{y}, \boldsymbol{\mu}(\tau)^{*}\right)=\int \pi\left(\boldsymbol{\phi}(\tau)^{*} \mid \mathbf{y}, \boldsymbol{\mu}(\tau)^{*}, \delta, \mathbf{P}(\tau), \mathbf{s}, \mathbf{v}\right) \pi\left(\delta, \mathbf{P}(\tau), \mathbf{s}, \mathbf{v} \mid \mathbf{y}, \boldsymbol{\mu}(\tau)^{*}\right) d \delta d \mathbf{P}(\tau) d \mathbf{s} d \mathbf{v}
$$

In order to obtain draws from the conditional distribution of $\delta, \mathbf{P}(\tau), \mathbf{s}, \mathbf{v}$ given $\mathbf{y}$ and $\boldsymbol{\mu}(\tau)^{*}$, we continue Gibbs sampling for an additional $N$ iterations with the complete conditional densities

$$
\begin{aligned}
& \pi\left(\mathbf{s}^{n} \mid \mathbf{P}(\tau)^{n-1}, \boldsymbol{\mu}(\tau)^{*}, \boldsymbol{\phi}(\tau)^{n-1}, \mathbf{v}^{n-1}, \delta^{n-1}, \mathbf{y}\right), \\
& \pi\left(\mathbf{P}(\tau)^{n} \mid \mathbf{s}^{n}, \boldsymbol{\mu}(\tau)^{*}, \boldsymbol{\phi}(\tau)^{n-1}, \mathbf{v}^{n-1}, \delta^{n-1}, \mathbf{y}\right), \\
& \pi\left(\boldsymbol{\phi}(\tau)^{n} \mid \mathbf{s}^{n}, \mathbf{P}(\tau)^{n}, \boldsymbol{\mu}(\tau)^{*}, \mathbf{v}^{n-1}, \delta^{n-1}, \mathbf{y}\right), \\
& \pi\left(\mathbf{v}^{n} \mid \mathbf{s}^{n}, \mathbf{P}(\tau)^{n}, \boldsymbol{\mu}(\tau)^{*}, \boldsymbol{\phi}(\tau)^{n}, \delta^{n-1}, \mathbf{y}\right), \\
& \pi\left(\delta^{n} \mid \mathbf{s}^{n}, \mathbf{P}(\tau)^{n}, \boldsymbol{\mu}(\tau)^{*}, \boldsymbol{\phi}(\tau)^{n}, \mathbf{v}^{n}, \mathbf{y}\right),
\end{aligned}
$$


where in each of these densities we condition upon $\boldsymbol{\mu}(\tau)^{*}$. The Monte Carlo estimate of $\pi\left(\boldsymbol{\phi}(\tau)^{*} \mid \mathbf{y}, \boldsymbol{\mu}(\tau)^{*}\right)$ is then found as

$$
\hat{\pi}\left(\boldsymbol{\phi}(\tau)^{*} \mid \mathbf{y}, \boldsymbol{\mu}(\tau)^{*}\right)=N^{-1} \sum_{n=1}^{N} \pi\left(\boldsymbol{\phi}(\tau)^{*} \mid \mathbf{y}, \boldsymbol{\mu}(\tau)^{*}, \delta^{n}, \mathbf{P}(\tau)^{n}, \mathbf{s}^{n}, \mathbf{v}^{n}\right),
$$

where $\left\{\delta^{n}, \mathbf{P}(\tau)^{n}, \mathbf{s}^{n}, \mathbf{v}^{n}\right\}_{n=1}^{N}$ are draws from the auxiliary Gibbs sampler.

This approach can be extended to obtain the estimates of the remaining ordinates in (23). Specifically, the ordinate $\pi\left(\delta(\tau)^{*} \mid \mathbf{y}, \boldsymbol{\mu}(\tau)^{*}, \boldsymbol{\phi}(\tau)^{*}\right)$ can be estimated as

$$
\hat{\pi}\left(\delta(\tau)^{*} \mid \mathbf{y}, \boldsymbol{\mu}(\tau)^{*}, \boldsymbol{\phi}(\tau)^{*}\right)=N^{-1} \sum_{i=1}^{N} \pi\left(\delta(\tau)^{*} \mid \mathbf{y}, \boldsymbol{\mu}(\tau)^{*}, \boldsymbol{\phi}(\tau)^{*}, \mathbf{P}(\tau)^{n}, \mathbf{s}^{n}, \mathbf{v}^{n}\right),
$$

where $\left\{\mathbf{P}(\tau)^{n}, \mathbf{s}^{n}, \mathbf{v}^{n}\right\}_{n=1}^{N}$ are an additional $N$ draws obtained by continuing the Gibbs sampler with the following conditional densities:

$$
\begin{aligned}
& \pi\left(\mathbf{s}^{n} \mid \mathbf{P}(\tau)^{n-1}, \boldsymbol{\mu}(\tau)^{*}, \boldsymbol{\phi}(\tau)^{*}, \mathbf{v}^{n-1}, \delta^{n-1}, \mathbf{y}\right), \\
& \pi\left(\mathbf{P}(\tau)^{n} \mid \mathbf{s}^{n}, \boldsymbol{\mu}(\tau)^{*}, \boldsymbol{\phi}(\tau)^{*}, \mathbf{v}^{n-1}, \delta^{n-1}, \mathbf{y}\right), \\
& \pi\left(\mathbf{v}^{n} \mid \mathbf{s}^{n}, \mathbf{P}(\tau)^{n}, \boldsymbol{\mu}(\tau)^{*}, \boldsymbol{\phi}(\tau)^{*}, \delta^{n-1}, \mathbf{y}\right), \\
& \pi\left(\delta^{n} \mid \mathbf{s}^{n}, \mathbf{P}(\tau)^{n}, \boldsymbol{\mu}(\tau)^{*}, \boldsymbol{\phi}(\tau)^{*}, \mathbf{v}^{n}, \mathbf{y}\right),
\end{aligned}
$$

which take $\boldsymbol{\mu}(\tau)^{*}$ and $\boldsymbol{\phi}(\tau)^{*}$ as fixed. Lastly for $\pi\left(\mathbf{p}_{i} .(\tau)^{*} \mid \mathbf{y}, \boldsymbol{\mu}(\tau)^{*}, \boldsymbol{\phi}(\tau)^{*}, \delta(\tau)^{*}\right), i=1, \ldots, K$, we use the estimates

$$
\hat{\pi}\left(\mathbf{p}_{i .}(\tau)^{*} \mid \mathbf{y}, \boldsymbol{\mu}(\tau)^{*}, \boldsymbol{\phi}(\tau)^{*}, \delta(\tau)^{*}\right)=N^{-1} \sum_{i=1}^{N} \pi\left(\mathbf{p}_{i .}(\tau)^{*} \mid \mathbf{y}, \boldsymbol{\mu}(\tau)^{*}, \boldsymbol{\phi}(\tau)^{*}, \delta(\tau)^{*}, \mathbf{P}(\tau)^{n}, \mathbf{s}^{n}, \mathbf{v}^{n}\right),
$$

where the auxiliary Gibbs draws $\left\{\mathbf{P}(\tau)^{n}, \mathbf{s}^{n}, \mathbf{v}^{n}\right\}_{n=1}^{N}$ are obtained by iteratively sampling from

$$
\begin{aligned}
& \pi\left(\mathbf{s}^{n} \mid \mathbf{P}(\tau)^{n-1}, \boldsymbol{\mu}(\tau)^{*}, \boldsymbol{\phi}(\tau)^{*}, \mathbf{v}^{n-1}, \delta^{*}, \mathbf{y}\right), \\
& \pi\left(\mathbf{P}(\tau)^{n} \mid \mathbf{s}^{n}, \boldsymbol{\mu}(\tau)^{*}, \boldsymbol{\phi}(\tau)^{*}, \mathbf{v}^{n-1}, \delta^{*}, \mathbf{y}\right), \\
& \pi\left(\mathbf{v}^{n} \mid \mathbf{s}^{n}, \mathbf{P}(\tau)^{n}, \boldsymbol{\mu}(\tau)^{*}, \boldsymbol{\phi}(\tau)^{*}, \delta^{*}, \mathbf{y}\right) .
\end{aligned}
$$

Upon substitution of all the estimated posterior ordinates into (23) we finally obtain from (13) the estimate of (the $\log$ of) the marginal likelihood, $\log \hat{\pi}(\mathbf{y} \mid \tau)$.

\section{Notes}

1 Farcomeni (2012) presents a similar algorithm for likelihood evaluation in the context of a latent Markov quantile regression model for longitudinal data.

2 As Chib (1995) explains, however, efficiency considerations dictate that $\log \pi(\mathbf{y} \mid \tau)$ is likely to be more accurately estimated at a high density point, such as the posterior mean or mode, rather than at a point in the tails of the posterior.

3 Such an approach based on maximizing the posterior probability $\operatorname{Pr}\left(s_{t} \mid \mathbf{y}\right)$ of each individual state $s_{t}, 1 \leq t \leq T$, is well known to maximize the number of correctly estimated states. In contrast, the Viterbi (1967) algorithm, which maximizes $\operatorname{Pr}\left(s_{1}, \ldots, s_{T} \mid \mathbf{y}\right)$, is suboptimal for individual state classification; see Lember and Koloydenko (2014).

4 Observe that the MADE criterion is quite natural in this context since the fundamental quantile estimation problem in (2) is defined via an absolute deviation loss function.

5 The MLE proceeds here by maximizing (5) subject to the stationary restriction in Assumption 2.

6 Koenker and Xiao (2006) fit a QAR model with $p=1$ lag to U.S. short-term interest rates.

\section{References}

Albert, ]., and S. Chib. 1993. "Bayes Inference Via Gibbs Sampling of Autoregressive Time Series Subject to Markov Mean and Variance Shifts." Journal of Business and Economic Statistics 11: 1-15.

Baur, D., T. Dimpfl, and R. Jung. 2012. “Stock Return Autocorrelations Revisited: A Quantile Regression Approach.” Journal of Empirical Finance 19: 254-265. 
Bauwens, L., A. Preminger, and ]. Rombouts. 2010. "Theory and Inference for a Markov Switching CARCH Model." Econometrics Journal 13: 218-244.

Billio, M., R. Casarin, and A. Osuntuyi. 2014. “Efficient Gibbs Sampling for Markov Switching GARCH Models." Computational Statistics and Data Analysis 100: 37-57.

Carter, C., and R. Kohn. 1994. “On Gibbs Sampling for State Space Models.” Biometrika 81: 541-553.

Casella, G., and E. George. 1992. “Explaining the Gibbs Sampler." American Statistician 46: 167-174.

Chen, C., R. Gerlach, B. Hwang, and M. McAleer. 2012. “Forecasting Value-at-Risk Using Nonlinear Regression Quantiles and the Intra-day Range." International Journal of Forecasting 28: 557-574.

Chib, S. 1995. "Marginal Likelihood from the Gibbs Output." Journal of the American Statistical Association 90: 1313-1321.

Chib, S. 1996. "Calculating Posterior Distributions and Modal Estimates in Markov Mixture Models." Journal of Econometrics 75: 79-97.

Chow, G. 1960. "Tests of Equality Between Sets of Coefficients in Two Linear Regressions." Econometrica 28: 591-605.

Christoffersen, P. 1998. “Evaluating Interval Forecasts." International Economic Review 39: 841-862.

Dagpunar, J. 1989. "An Easily Implemented Generalized Inverse Gaussian Cenerator." Communications in Statistics - Simulation and Computation 18: 703-710.

Engle, R., and S. Manganelli. 2004. “CAViaR: Conditional Autoregressive Value at Risk by Regression Quantiles.” Journal of Business and Economic Statistics 22: 367-381.

Escanciano, J., and C. Velasco. 2010. “Specification Tests of Parametric Dynamic Conditional Quantiles." Journal of Econometrics 159: 209221.

Farcomeni, A. 2012. “Quantile Regression for Longitudinal Data Based on Latent Markov Subject-Specific Parameters.” Statistics and Computing 22: 141-152.

Ferreira, M. 2011. “Capturing Asymmetry in Real Exchange Rate with Quantile Autoregression." Applied Economics 43: 327-340.

Frühwirth-Schnatter, S. 2001. "Markov Chain Monce Carlo Estimation of Classical and Dynamic Switching and Mixture Models." Journal of the American Statistical Association 96: 194-209.

Frühwirth-Schnatter, S. 2006 Finite Mixture and Markov Switching Models New York, NY: Springer.

Gaglianone, W., L. Lima, O. Linton, and D. Smith. 2011. "Evaluating Value-at-Risk Models Via Quantile Regression." Journal of Business and Economic Statistics 29: 150-160.

Galvao, A., G. Montes-Rojas, and J. Olmo. 2011. “Threshold Quantile Autoregressive Models.” Journal of Time Series Analysis 32: $253-267$.

Galvao, A., G. Montes-Rojas, and S. Park. 2013. "Quantile Autoregressive Distributed Lag Model with an Application to House Price Returns." Oxford Bulletin of Economics and Statistics 75: 307-321.

Garcia, R., and P. Perron. 1996. "An Analysis of the Real Interest Rate Under Regime Shifts." Review of Economics and Statistics 78: 111-125.

Celfand, A., A. Smith, and T.-M. Lee. 1992. "Bayesian Analysis of Constrained Parameter and Truncated Data Problems Using Cibbs Sampling." Journal of the American Statistical Association 87: 523-532.

Geraci, M., and M. Bottai. 2007. “Quantile Regression for Longitudinal Data Using the Asymmetric Laplace Distribution.” Biostatistics 8: 140-154.

Cerlach, R., C. Chen, and N. Chan. 2011. "Bayesian Time-Varying Quantile Forecasting for Value-at-Risk in Financial Markets." Journal of Business and Economic Statistics 29: 481-492.

Geweke, J. 1992. "Evaluating the Accuracy of Sampling-Based Approaches to Calculating Posterior Moments."; In Bayesian Statistics, edited by J. Bernardo, J. Berger, A. Dawid, and A. Smith, Vol. 4 169-193. Oxford: Oxford University Press.

Coldfeld, S., and R. Quandt. 1973. “A Markov Model for Switching Regressions.” Journal of Econometrics 1: 3-16.

Hamilton, J. 1989. "A New Approach to the Economic Analysis of Non Stationary Time Series and the Business Cycle." Econometrica 57: 357384.

Kass, R., and A. Raftery. 1995. “Bayes Factors." Journal of the American Statistical Association 90: 773-795.

Kim, C.-J., and C. Nelson. 1999 State-Space Models with Regime Switching: Classical and Gibbs-Sampling Approaches with Applications Cambridge, MA: MIT Press.

Koenker, R., and G. Bassett. 1978. “Regression Quantiles.” Econometrica 46: 33-49.

Koenker, R., and J. Machado. 1999. “Coodness of Fit and Related Inference Processes for Quantile Regression." Journal of the American Statistical Association 94: 1296-1310.

Koenker, R., and Z. Xiao. 2006. “Quantile Autoregession.” Journal of the American Statistical Association 101: 980-990.

Kottas, A., and M. Krnjajić. 2009. “Bayesian Semiparametric Modelling in Quantile Regression." Scandinavian Journal of Statistics 36: 297319.

Kotz, S., T. Kozubowski, and K. Podgórski. 2001 The Laplace Distribution and Generalizations: A Revisit with Applications to Communications, Economics, Engineering, and Finance Boston: Birkhäuser.

Kozumi, H., and G. Kobayashi. 2011. "Cibbs Sampling Methods for Bayesian Quantile Regression." Journal of Statistical Computation and Simulation 81: 1565-1578.

Kupiec, P. 1995. “Techniques for Verifying the Accuracy of Risk Measurement Models." Journal of Derivatives 3: 73-84.

Lee, C.-C., C.-F. Lee, and C.-C. Lee. 2014. "Asymmetric Dynamics in REIT Prices: Further Evidence Based on Quantile Regression Analysis." Economic Modelling 42: 29-37.

Lember, J., and A. Koloydenko. 2014. "Bridging Viterbi and Posterior Decoding: A Ceneralized Risk Approach to Hidden Path Inference Based on Hidden Markov Models." Journal of Machine Learning Research 15: 1-58.

Manzan, S. 2015. "Forecasting the Distribution of Economic Variables in a Data-Rich Environment." Journal of Business and Economic Statistics 33: 144-164.

McCulloch, R., and R. Tsay. 1994. "Statistical Analysis of Economic Time Series Via Markov Switching Models." Journal of Time Series Analysis 15: 523-539.

Piger, ]. 2009. "Econometrics: Models of Regime Changes."; In Encyclopedia of Complexity and Systems Science, edited by Robert A. Meyers, 190-202. New York, NY: Springer. 
Qu, Z. 2008. “Testing for Structural Change in Regression Quantiles.” Journal of Econometrics 146: 170-184.

Scott, S. 2002. "Bayesian Methods for Hidden Markov Models: Recursive Computing in the 21st Century." Journal of the American Statistical Association 97: 337-351.

Tierney, L. 1994. “Markov Chains for Exploring Posterior Distributions.” Annals of Statistics 22: 1701-1728.

Tong, H. 1983 Threshold Models in Non-linear Time Series Analysis New York, NY: Springer-Verlag.

Tsionas, E. 2003. “Bayesian Quantile Inference." Journal of Statistical Computation and Simulation 9: 659-674.

Viterbi, A. 1967. "Error Bounds for Convolutional Codes and an Asymptotically Decoding Algorithm." IEEE Transactions on Information Theory 13: $260-269$.

Wu, Y., and Y. Liu. 2009. “Stepwise Multiple Quantile Regression Estimation Using Non-crossing Constraints.” Statistics and its Interface 2: 299-310.

Yang, Z., A. Tu, and Y. Zeng. 2014. “Dynamic Linkages Between Asian Stock Prices and Exchange Rates: New Evidence from Causality in Quantiles." Applied Economics 46: 1184-1201.

Ye, W., Y. Zhu, Y. Wu, and B. Miao. 2016. “Markov Regime-Switching Quantile Regression Models and Financial Contagion Detection.” Insurance: Mathematics and Economics 67: 21-26.

Yu, K., and R. Moyeed. 2001. “Bayesian Quantile Regression.” Statistics and Probability Letters 54: 437-447.

Yue, Y., and H. Rue. 2011. "Bayesian Inference for Additive Mixed Quantile Regression Models." Computational Statistics and Data Analysis 55: 84-96.

Supplemental Material: The online version of this article offers supplementary material (DOI: https://doi.org/10.1515/snde-2016-0078). 